\title{
Emission-Line Properties of the Optical Filaments of NGC 1275
}

\author{
Bassem M. Sabra \& Joseph C. Shields \\ Department of Physics \& Astronomy, Ohio University, Athens, OH 45701 \\ and \\ Alexei V. Filippenko \\ Department of Astronomy, University of California, Berkeley, CA 94720-3411
}

\begin{abstract}
Extended nebular filaments are seen at optical wavelengths in NGC 1275, the central galaxy in the Perseus cluster. The agents responsible for the excitation of these filaments remain poorly understood. In this paper we investigate possible mechanisms for powering the filaments, using measurements from an extensive spectroscopic data set acquired at the Lick Observatory 3-m Shane telescope. The results show that the filaments are in an extremely low ionization and excitation state. The high signal-to-noise ratio of the spectra allows us to measure or place sensitive upper limits on weak but important diagnostic lines. We compare the observed line intensity ratios to the predictions of various ionization models, including photoionization by an active galactic nucleus, shock heating, stellar photoionization, and photoionization by the intracluster medium. We also investigate possible roles for cluster extreme-ultraviolet emission, and filtering of cluster soft X-ray emission by an ionized screen, in the energetics of the filaments. None of these mechanisms provides an entirely satisfactory explanation for the physical state of the nebulae. Heating and ionization by reconnection of the intracluster magnetic field remains a potentially viable alternative, which merits further investigation through Faraday rotation studies.
\end{abstract}

Subject headings: galaxies: clusters: individual (Perseus) - cooling-flows galaxies: individual (NGC 1275) — galaxies: ISM

\section{INTRODUCTION}

Extended nebular filaments are commonly seen in association with the central galaxies of galaxy clusters. Clusters that feature a massive central galaxy are also often identified 
as having a cooling flow, based on cooling-timescale arguments or other evidence (Fabian, Nulsen, \& Canizares 1984). This association has prompted speculation that the optical nebulosity is linked causally or energetically to the intracluster medium (ICM), and hence may be an important diagnostic of the cooling-flow phenomenon. As a result, several previous studies have investigated optical "cooling-flow filaments" in attempts to understand their physical properties. These efforts have helped delineate the observed characteristics of the nebulosity, and have prompted a variety of theoretical explanations for what is seen.

Optical spectroscopy reveals that the filaments emit strongly in low-ionization forbidden lines, thus resembling Low Ionization Nuclear Emission-Line Regions (LINERs; Heckman 1980). Heckman et al. (1989) also reported evidence that cooling-flow filaments can be separated into two characteristic groups based on their line-intensity ratios. Typical ratios for Type I systems are $[\mathrm{N}$ II $] \lambda 6583 / \mathrm{H} \alpha \approx 2,[\mathrm{~S} \mathrm{II}] \lambda 6717 / \mathrm{H} \alpha \approx 0.7$, and $[\mathrm{O} \mathrm{I}] \lambda 6300 / \mathrm{H} \alpha \approx 0.2$; these objects tend to have low X-ray and $\mathrm{H} \alpha$ luminosities and are associated with low cooling rates and small optical filaments. In contrast, typical ratios for Type II objects are $[\mathrm{N}$ II $] \lambda 6583 / \mathrm{H} \alpha \approx 1,[\mathrm{~S} \mathrm{II}] \lambda 6717 / \mathrm{H} \alpha \approx 0.4$, and $[\mathrm{O} \mathrm{I}] \lambda 6300 / \mathrm{H} \alpha \approx 0.3$; these sources have high $\mathrm{X}$-ray and $\mathrm{H} \alpha$ luminosities and are associated with high inferred cooling rates and extended optical filaments. Theoretical efforts to interpret the optical spectra have invoked various combinations of cooling or irradiation from the ICM, shocks, active galactic nucleus (AGN) emission or outflows, magnetic reconnection, and hot stars. However, none of these scenarios has proven entirely satisfactory in explaining the observations; consequently, the origins and energetics of these systems remain poorly understood.

An important example of optical filaments in apparent association with a cooling flow is found in NGC 1275 (Kent \& Sargent 1979), the central elliptical galaxy in the Perseus cluster (Abell 426). The nebular gas extends to at least $20 \mathrm{kpc}$ from the center of the galaxy (assuming $\mathrm{H}_{0}=75 \mathrm{~km} \mathrm{~s}^{-1} \mathrm{Mpc}^{-1}$ ), with a complicated spatial structure; the optical spectrum fits within Heckman et al.'s Type II category. The surrounding intracluster medium is believed to have a cooling flow, with mass deposition rates of up to $130 \mathrm{M}_{\odot} \mathrm{yr}^{-1}$ (Fabian, Cowie, \& Grindley 1981; Allen et al. 1999). NGC 1275 features additional unusual characteristics that may be relevant to interpreting the extended nebular emission. The central elliptical galaxy is seen at a recession velocity of $\sim 5200 \mathrm{~km} \mathrm{~s}^{-1}$, but is partially obscured by a smaller foreground galaxy at a velocity of $\sim 8200 \mathrm{~km} \mathrm{~s}^{-1}$ (Minkowski 1957); the redshift difference is consistent with the velocity dispersion observed for this cluster (e.g., Kent \& Sargent 1983). NGC 1275 is also host to a strong AGN and associated radio source, 3C 84, which emits jets that may be interacting with the ambient gas (Pedlar, Booler, \& Davies 1983; Pedlar et al. 1990).

In this paper, we report the results of a detailed study of the physical properties of the 
optical filaments associated with NGC 1275. This work makes use of extensive spectroscopic observations to quantify two-dimensional aspects of the observed emission, and also places sensitive limits on weak spectral features of diagnostic interest. The results can be used to place stringent limits on the role of various agents proposed for heating or ionization of this plasma.

\section{Observations and Data Reduction}

Long-slit spectra of the filaments of NGC 1275 were acquired in 10 observing runs during 1988 - 1990, using the Shane 3-m telescope + UV Schmidt CCD spectrograph at Lick Observatory (Miller \& Stone 1987). The resulting data cover the wavelength range 3396 $6940 \AA$ in three bandpasses, spanning $3396-5000 \AA, 4695-6280 \AA$, and $6130-6940 \AA$. The slit was $2.5^{\prime \prime}$ wide and $132^{\prime \prime}$ long, with spatial sampling of $0.66^{\prime \prime}$ pixel $^{-1}$. Observations were acquired with the slit centered on the nucleus and oriented at four different position angles $\left(\mathrm{PA}=62^{\circ}, 77^{\circ}, 283^{\circ}\right.$, and $\left.313^{\circ}\right)$ selected to lie along filaments with the highest surface brightness in $\mathrm{H} \alpha$ (see Figure 1). Typical atmospheric seeing was $2^{\prime \prime}$ and not all observations were done under strictly photometric conditions. The spectral resolution was $\sim 3.5 \AA$ longward of $6130 \AA$ and $\sim 7 \AA$ elsewhere. The giant H II region detected by Shields \& Filippenko (1990a) lies at $\mathrm{PA}=62^{\circ}$. Line emission from the high-velocity system $(c z \approx 8200$ $\mathrm{km} \mathrm{s}^{-1}$ ) appears primarily northwest of the nucleus (e.g., Unger et al. 1990); it is visible in our spectra acquired at $\mathrm{PA}=313^{\circ}$, and to a lesser extent at $\mathrm{PA}=283^{\circ}$.

The data were reduced using standard techniques, as implemented in IRAF 円. Spectra acquired at the same position angle and wavelength setting were averaged after flux and wavelength calibration and sky subtraction. Estimates of the sky contribution were derived from regions near the ends of the slit, where no nebular emission was evident and the contribution of galaxy starlight was minimal. Measurements of filament emission were obtained for regions where $\mathrm{H} \alpha$ emission was detected in a visual inspection of the 2-dimensional (2-D) spectra.

Emission-line strengths for the stronger lines were measured along the slit on a pixel-bypixel basis, using an automated algorithm. For each resulting 1-D spectrum, the continuum in each wavelength setting was approximated by a low-order polynomial fit to emission-free bandpasses. After subtraction of the continuum, candidate emission lines were measured

\footnotetext{
${ }^{1}$ The Image Reduction and Analysis Facility (IRAF) is distributed by the National Optical Astronomy Observatories, which is operated by the Association of Universities for Research in Astronomy, Inc. (AURA), under cooperative agreement with the National Science Foundation.
} 
by fitting Gaussian profiles to all features detected at $\geq 3 \sigma$ significance. The lines were subsequently identified, and spurious features rejected, by comparison of the measured list with an input list of line wavelengths, shifted by an a priori estimate of the emission redshift; this redshift value was measured interactively for $\mathrm{H} \alpha$ in the spectrum of the filament coadded along the slit. Lines were identified if the predicted and observed wavelengths agreed to within $5 \AA$. This bound was set to allow for the correct identification of lines in the presence of substantial $\left(\leq 300 \mathrm{~km} \mathrm{~s}^{-1}\right.$ ) velocity gradients that are observed in this gas (e.g., Rubin et al. 1978). The line flux measured by this method is $\sqrt{2 \pi} \sigma_{\lambda} F_{p}$, where $\sigma_{\lambda}$ is the line profile dispersion as determined by the Gaussian fit and $F_{p}$ is the peak height of the emission line above the continuum.

Estimation of line-intensity ratios for the NGC 1275 nebulosity requires correction for reddening. The filaments are subject to a Galactic foreground extinction of $A_{V}=0.82$ mag (Lockman \& Savage 1995) as well as internal extinction, some of which may be due to the high-velocity system. The limited signal-to-noise ratio $(\mathrm{S} / \mathrm{N})$ of many of the pixel-bypixel spectral extractions translates into significant uncertainties in a reddening value based on Balmer-line ratios, and as a result we derived an average reddening correction for each filament (i.e., region of nebular emission seen on one side of the nucleus at a given PA). We obtained total extinction values from the measured $\mathrm{H} \alpha / \mathrm{H} \beta$ line-intensity ratio, assuming an intrinsic value of 2.86 (Hummer \& Storey 1987) and the extinction curve of Cardelli, Clayton, \& Mathis (1989). The results were compared with extinction values implied by line ratios employing $\mathrm{H} \gamma$ and $\mathrm{H} \delta$, and were found to be consistent, with the exception of measurements for $\mathrm{PA}=313^{\circ}$, where the limited $\mathrm{S} / \mathrm{N}$ made the high-order Balmer lines unmeasurable, and for $\mathrm{PA}=283^{\circ}$. The line ratios at the latter PA show evidence of underlying stellar absorption, which is not surprising in light of earlier reports of early-type stellar contributions to the integrated light seen in some portions of NGC 1275 (e.g., Wirth et al. 1983). The influence of the stellar features is apparently strongest at this PA since the emission equivalent widths are relatively weak. For this filament we determined a consistent reddening value for the higher Balmer transitions subject to absorption with constant equivalent width. The total extinctions (including the Galactic contribution, and neglecting the redshift difference of the absorbers) calculated by these means are $A_{V}=1.0 \pm 0.2,0.8 \pm 0.2,2.3 \pm 0.5$, and $1.1 \pm 0.4 \mathrm{mag}$ for filaments at $\mathrm{PA}=62^{\circ}, 77^{\circ}, 283^{\circ}$, and $313^{\circ}$, respectively. The larger value of extinction towards $\mathrm{PA}=283^{\circ}$ may be caused by the high-velocity system (Unger et al. 1990). The B-band atmospheric feature appears slightly longward of redshifted [S II] $\lambda 6731$, but does not affect measurements of that line.

In addition to studying the spatially resolved emission at each PA, we generated a composite spectrum of the filament system in order to study weak emission features. For each PA, the 2-D spectra were averaged over intervals of high $\mathrm{S} / \mathrm{N}$ filament emission $(\mathrm{S} / \mathrm{N}$ 
$\gtrsim 5$ for $\mathrm{H} \alpha$ ). For $\mathrm{PA}=62^{\circ}$, this criterion translated into an extracted region $14^{\prime \prime}$ wide, centered $23^{\prime \prime}$ away from the nucleus. The $\mathrm{PA}=77^{\circ}$ and $\mathrm{PA}=283^{\circ}$ extractions were $18.5^{\prime \prime}$ wide, centered at $21^{\prime \prime}$ and $20^{\prime \prime}$ away from the nucleus, respectively. The extraction for PA $=313^{\circ}$ spanned $33^{\prime \prime}$, centered at $26^{\prime \prime}$ from the nucleus; we ultimately decided not to include this extraction in the composite spectrum because the emission-line equivalent widths were generally less than for the other PAs, with a correspondingly lower S/N. The resulting 1D spectra were shifted to the rest wavelength scale, as determined from the redshift of the Balmer lines, and the spectra for different PAs were then combined to a single overall average. The relative velocity shifts between the filament extractions were less than $90 \mathrm{~km} \mathrm{~s}^{-1}$. The final composite spectrum (Figure 2) represents a total integration time of 10 hours in each wavelength setting.

In order to improve the accuracy of the continuum subtraction for the composite spectrum, we experimented with modeling the stellar continuum with templates derived from observations of emission-free galaxies. A reasonable correspondence in terms of the strength of stellar features in NGC 1275 was found with the spectrum of NGC 205. The stellar velocity dispersion of NGC 1275 is $250 \mathrm{~km} \mathrm{~s}^{-1}$ (Nelson \& Whittle 1995) while that of NGC 205 is 50 $\mathrm{km} \mathrm{s}^{-1}$ (Held, Mould, \& de Zeeuw 1990). We consequently convolved the NGC 205 spectrum with a Gaussian having $\sigma=245 \mathrm{~km} \mathrm{~s}^{-1}$ in order to match the dispersion seen in NGC 1275. To optimize the template match, a fit with $\chi^{2}$ minimization was carried out using SPECFIT as implemented in IRAF (Kriss 1994), varying the template redshift, scaling, and reddening. A best fit was obtained with $A_{V}=0.6 \pm 0.1 \mathrm{mag}$. The Galactic extinction for NGC 205 is $A_{V}=0.10 \mathrm{mag}$ (Burstein \& Heiles 1984); if the two galaxies were perfectly matched in spectral properties, this would imply a total extinction for NGC 1275 of $A_{V}=0.7 \pm 0.1$ mag, in agreement with the Galactic foreground estimate. This value is somewhat less than is obtained from Balmer emission lines for the filaments; the disparity may stem from real differences in the reddening of starlight and nebular emission, and/or differences in stellar populations for NGC 1275 and NGC 205. The spectral regions over which we performed the continuum fitting do not appear to be affected strongly by metallicity differences between NGC 1275 and NGC 205.

For the stronger emission features, line strengths measured after subtraction of this continuum fit were consistent with the results obtained from a smooth fit to the continuum, as employed for the pixel-by-pixel extractions. Use of the galaxy template was important for obtaining accurate values or limits for the weaker features, however, and for some lines the template fit was locally optimized (by adjusting the scaling) in order to match the observed continuum. The line fluxes obtained after subtraction of the continuum template are listed in Table 1, and were obtained from Gaussian profile fits as described above. Error bars listed for the detected lines represent the root-mean-square uncertainty per pixel $\left(\sigma_{c}\right)$ 
in the local continuum, summed in quadrature over a width of $6 \sigma_{\lambda}$, i.e., $\sqrt{6} \sigma_{c} \sigma_{\lambda}$. For lines that were not detected, we list $3 \sigma$ upper limits of $\sqrt{2 \pi} \sigma_{\lambda}\left(3 \sigma_{c}\right)$, where $\sigma_{\lambda}$ is the width of either $\mathrm{H} \beta$ or $\mathrm{H} \alpha$, depending on which of these Balmer lines is closer in wavelength. For the composite spectrum, the $\mathrm{H} \alpha / \mathrm{H} \beta$ ratio implies a total extinction of $A_{V}=1.21$ mag, yielding an intrinsic value of $A_{V}=0.39 \mathrm{mag}$. The higher-order Balmer lines in the continuum-subtracted spectrum showed evidence for being over-corrected for the underlying stellar absorption: $(\mathrm{H} \delta / \mathrm{H} \beta)_{\text {Observed }}>(\mathrm{H} \delta / \mathrm{H} \beta)_{\text {Case } B}$. The implied disparity between the NGC 1275 and template spectra does not significantly influence the estimate of $A_{V}$ obtained from $\mathrm{H} \alpha / \mathrm{H} \beta$, however, due to the large equivalent widths of these emission features.

\section{General Results}

\subsection{Line-Ratio Diagrams}

An informative overview of the emission properties of the NGC 1275 filaments can be obtained from standard line-ratio diagrams employed for nebular classification (Baldwin, Phillips, \& Terlevich 1981; Veilleux \& Osterbrock 1987). Figure 3 shows three such diagrams along with measurements from the pixel-by-pixel extracted spectra for NGC 1275 . The observations have been corrected for reddening, as described in $\S 2$, and the use of intensity ratios of lines close in wavelength makes these diagrams insensitive to any residual reddening uncertainties. Loci commonly adopted for classification of H II regions, Seyfert nuclei, and LINERs are also indicated (e.g., Shields \& Filippenko 1990b).

As found in previous work, the filaments in NGC 1275 are characterized by low-ionization emission, resembling that of LINERs (e.g., Heckman et al. 1989). An additional diagram illustrating the ionization state of the gas is shown in Figure 4, which is a plot of $[\mathrm{O} \mathrm{I}] \lambda 6300 /[\mathrm{O} \mathrm{III}] \lambda 5007$ versus $[\mathrm{O} \mathrm{II}] \lambda 3727 /[\mathrm{O} \mathrm{III}] \lambda 5007$; this combination is more sensitive to reddening, but has the benefit of tracing the ionization of a single element. Figure 4 shows that the filaments overlap the low-ionization extreme of LINER behavior, and in particular extend to very high $[\mathrm{O}$ I] $/[\mathrm{O}$ III $]$ ratios. We note further that in Figure 3 the measurements do not uniformly populate the LINER locus, but instead are clearly concentrated to relatively low values for each of the forbidden-line/recombination-line ratios. Another way of stating this result is that the nebulosity is not merely described by low ionization, as for LINERs, but is also characterized by low excitation. Since the forbidden lines arise from collisional excitation, while the Balmer lines trace recombination, this result implicates a relatively low degree of heating per ionization in the cluster filaments.

An interesting result illustrated by the 2-D spectra is that the distribution of points 
shows a larger variation in the $[\mathrm{O} \mathrm{III}] / \mathrm{H} \beta$ ratio than in ratios employing the forbidden lines of lower ionization, such as $[\mathrm{N} \mathrm{II}] / \mathrm{H} \alpha$, [S II $] / \mathrm{H} \alpha$, and $[\mathrm{O} \mathrm{I}] / \mathrm{H} \alpha$. The measurements include data for $\mathrm{PA}=62^{\circ}$ at the location of a giant $\mathrm{H}$ II region reported by Shields \& Filippenko (1990a). The ratios for this locale are distinct from those elsewhere in the filaments, and contribute excursions to low values of $[\mathrm{O} \mathrm{I}] / \mathrm{H} \alpha$ and $[\mathrm{S} \mathrm{II}] / \mathrm{H} \alpha$ seen in Figures $3 b$ and $3 c$. Removal of these points would increase the contrast between variation in $[\mathrm{O} \mathrm{III]} / \mathrm{H} \beta$ and in the other ratios.

\subsection{Coronal Emission And CoOling}

A set of weak lines that are of special interest are the coronal lines. These high-ionization collisionally excited lines are emitted from gas at a temperature of $\sim 10^{5.5-6.3} \mathrm{~K}$, which is intermediate between that of the ICM, $10^{6-7} \mathrm{~K}$, and the optical filaments, $\sim 10^{4} \mathrm{~K}$. The coronal lines trace gas in the process of cooling and their strength is a direct measure of the mass cooling rate, $\dot{M}$ (Hu, Cowie, \& Wang 1985; Sarazin \& Graney 1991). Detection of coronal lines may thus provide important diagnostics of the cooling-flow phenomenon.

The wavelength range covered in our spectra includes several coronal lines, specifically $[\mathrm{Ni} \mathrm{XII}] \lambda 4232,[\mathrm{Ar} \mathrm{XIV}] \lambda 4414,[\mathrm{Fe} \mathrm{XIV}] \lambda 5303,[\mathrm{Ca} \mathrm{XV}] \lambda 5445,[\mathrm{Fe} \mathrm{X}] \lambda 6374$, and $[\mathrm{Ni} \mathrm{XII}] \lambda 6702$. None of these transitions was detected in our composite spectrum. Upper limits to these lines were measured after subtracting a fit to the stellar continuum using a galaxy template of NGC 205, as described in $\S 2$. The location of [Fe X] $] 6374$ coincides with the red wing of $[\mathrm{O} \mathrm{I}] \lambda 6364$ and consequently warranted special treatment. The following procedure was used to remove the oxygen line. A stellar continuum was fitted over the wavelength range of 6250 to $6350 \AA$ as described in $\S 2$, simultaneously with a Gaussian to $[\mathrm{O} \mathrm{I}] \lambda 6300$. To remove $[\mathrm{O} \mathrm{I}] \lambda 6364$, we divided the template of $[\mathrm{O} \mathrm{I}] \lambda 6300$ by the line ratio of 3.0 dictated by atomic parameters (Osterbrock 1989), and subtracted the resulting profile and continuum fit from the composite spectrum. An upper limit for [Fe X] $\lambda 6374$ was derived from the residual. The upper limits on the coronal lines were corrected for extinction corresponding to $A_{V}=1.2 \mathrm{mag}$, the same as that applied to the detected lines. The results are listed in Table 2.

Utilizing theoretical models and our upper limits, we can calculate upper limits on the cooling rate of the hot ICM as measured in our apertures. The coronal line expected to have the highest emissivity is [Fe X] $\lambda 6374$, for which Sarazin \& Graney (1991) predict $1.39 \times 10^{37} \mathrm{erg} \mathrm{s}^{-1}$ for every $1 \mathrm{M}_{\odot} \mathrm{yr}^{-1}$. For the one-pixel equivalent aperture corresponding to the composite spectrum, the upper limit to the reddening-corrected surface brightness of this line that we measure is $\sim 2.7 \times 10^{-17} \mathrm{erg} \mathrm{s}^{-1} \mathrm{~cm}^{-2} \operatorname{arcsec}^{-2}$. The composite spectrum 
is averaged over regions that extend out to a radius of $\sim 28^{\prime \prime}$. Assuming that the coronal emission is distributed uniformly within this radius, our measurement suggests an upper limit for the luminosity of $[\mathrm{Fe} \mathrm{X}]$ of $\sim 4.0 \times 10^{40} \mathrm{erg} \mathrm{s}^{-1}$, corresponding to a mass cooling rate of less than $2900 \mathrm{M}_{\odot} \mathrm{yr}^{-1}$. This value of $\dot{M}$ is well above the accretion rate derived from X-ray observations; upper limits to the other coronal features would lead to even less restrictive limits on $\dot{M}$. These results underscore the practical difficulty in using the coronal lines to constrain $\dot{M}$, even with the investment of substantial amounts of observing time.

The strength of coronal emisson relative to $\mathrm{H} \alpha$ is of interest if the nebular filaments are energetically coupled to the cooling ICM. A cold filament irradiated by an isobarically cooling gas reprocesses the ionizing radiation into $\mathrm{H} \alpha$ with an efficiency $~ 3 \%$ (Voit \& Donahue 1990; Donahue \& Voit 1991). The H $\alpha$ strength would thus be $0.03(5 / 2)\left(k T / \mu m_{p}\right) \dot{M}$, where $m_{p}$ is the proton mass, $\mu$ is the mean mass per particle in units of $m_{p}, T$ is the temperature of the hot gas, and $k$ is the Boltzmann constant. In this picture, both the $\mathrm{H} \alpha$ and coronal-line luminosities scale with $\dot{M}$, leading to well-defined predictions for their ratios, which we list in Table 3 (Voit, Donahue, \& Slavin 1994; Sarazin \& Graney 1991).

The observed upper limits to the coronal-line strengths relative to $\mathrm{H} \alpha$ are larger than those of the predictions, thus leaving open the possibility that the filaments are ionized by the cooling ICM, a scenario we examine in more detail in Section 4. The same data set as employed here was used by Shields \& Filippenko (1992) to obtain a more restrictive limit on $[\mathrm{Fe} \mathrm{X}] \lambda 6374$, after binning the data on the basis of $\mathrm{H} \alpha$ surface brightness. The use of an intermediate surface brightness bin produced a somewhat higher $\mathrm{S} / \mathrm{N}$ ratio in the continuum. Their upper limit to $[\mathrm{Fe} \mathrm{X}] \lambda 6374 / \mathrm{H} \alpha$ was $1.4 \times 10^{-3}$, adjusted to use the same upper-limit definition and reddening correction employed here. This value is $\sim 36 \%$ less than the theoretical prediction, which may implicate other ionization sources for the filaments. However, this conclusion assumes that the cooling plasma irradiating the nebular gas lies within our measurement aperture, rather than on larger scales, and that the coronal feature displays the same velocity width as $\mathrm{H} \alpha$. Similar observational upper limits for coronal lines from cooling-flow filaments in other clusters have been reported by Donahue \& Stocke (1994) and Yan \& Cohen (1995).

An additional complication to the use of the coronal lines as diagnostics of cooling stems from persistent uncertainties in the relevant atomic data (e.g., Mohan, Hibbert, \& Kingston 1994; Pelan \& Berrington 1995; Storey, Mason, \& Saraph 1996; Ferguson, Korista, \& Ferland 1997; Oliva 1997). As summarized by Voit et al. (1994) for [Fe XIV] 75303 , alternative choices of atomic parameter values can lead to order-of-magnitude differences in the predicted emissivities for a cooling ICM. As noted in Table 3, our upper limit to $[\mathrm{Fe} \mathrm{XIV}] \lambda 5303 / \mathrm{H} \alpha$ is less than the value predicted by Voit et al. (1994) but larger than 
that of Sarazin \& Graney (1991). An unambiguous interpretation of coronal-line limits or detections will require further improvement in the atomic data.

\subsection{IONIZATION MECHANISMS}

A question central to the understanding of cooling-flow filaments is the issue of what ionizes and powers these systems. A comparison of the recombination expected for mass deposition rates obtained from X-ray observations with the observed $\mathrm{H} \alpha$ line luminosities makes it clear that simple cooling of the gas with a transition through a nebular phase is insufficient to explain the energy requirements of the filaments in NGC 1275 and other objects (e.g., Heckman et al. 1989). A variety of mechanisms have been proposed for the ionization of cooling-flow filaments. Below we compare the predictions of these scenarios with the results of our observational study, taking into account various physical constraints.

\subsubsection{IONIZATION BY AN AGN}

NGC 1275 shows evidence for a Seyfert-like nucleus (Seyfert 1943), as is clear from the emission-line spectrum (e.g., Filippenko \& Sargent 1985) and nonstellar continuum from the central regions. If the extended nebulosity is photoionized by this continuum, the ionization state of the gas will reflect the ionization parameter, which we define here as the ratio of ionizing photon and electron densities at the face of the irradiated cloud. The line ratio $[\mathrm{S} \mathrm{II}] \lambda 6717 /[\mathrm{S} \mathrm{II}] \lambda 6731$ is sensitive to the electron density, but remains consistent with the low-density limit $\left(n_{e} \lesssim 10^{2} \mathrm{~cm}^{-3}\right.$; Osterbrock 1989) throughout the filaments. While the density may vary below this threshold within the measured regions, pressure arguments suggest that large fluctuations are unlikely (e.g., Johnstone \& Fabian 1988; Heckman et al. 1989). If the nebular filaments are ionized by the AGN, we would then expect that the ionization parameter would largely vary in the same way as the ionizing flux, proportional to $r^{-2}$. The line ratio that is the best diagnostic of the ionization state is $[\mathrm{O}$ III $] \lambda 5007 / \mathrm{H} \beta$, since this ratio involves a high-ionization line (e.g., Ferland \& Netzer 1983; Ho et al. 1993). The filaments range from $\sim 10^{\prime \prime}$ to $\sim 30^{\prime \prime}$ in projected distance from the nucleus. If these structures lie in the plane of the sky, then the change in the ionization parameter due to geometric dilution of flux would be approximately one order of magnitude, translating into a significant variation in $[\mathrm{O}$ III $] / \mathrm{H} \beta$. Values of this line ratio as a function of radius for each

of the filaments are shown in Figure 5. While negative gradients are seen in some places, the variations at large radial distance tend to be small and/or non-monotonic, a result that is in agreement with previous observations having more limited PA coverage (Johnstone \& 
Fabian 1988).

The observed behavior may still be consistent with photoionization by a central source if the physical distance from the nucleus is not indicated by the projected distance. Johnstone \& Fabian (1988) constructed a detailed model to study the effects of geometry in the filaments of NGC 1275, and were able to reproduce the observed line ratios as a function of projected distance, but suggested some disagreement with the observed surface brightness of the filaments. The present data show reasonable consistency between measured surface brightnesses and predictions for ionization by the nucleus. The flux in the ultraviolet continuum measured by the International Ultraviolet Explorer (Kinney et al. 1991) and X-ray measurements from Einstein (Branduardi-Raymont et al. 1981) imply a 2-point spectral index between $2500 \AA$ (after dereddening) and $2 \mathrm{keV}$ of $\alpha_{o x}=-1.4$ (assuming $f_{\nu} \propto \nu^{\alpha}$ ). Extrapolating the UV flux assuming a power law with this slope implies an ionizing luminosity of $2 \times 10^{54}$ photons $\mathrm{s}^{-1}$. Clouds with a density of $n_{e}=100 \mathrm{~cm}^{-3}$ at a characteristic distance of $\sim 7 \mathrm{kpc}$ will be described by an ionization parameter of $U \approx 10^{-4}$, consistent with the observed line ratios. These numbers also predict for ionization-bounded clouds an emission measure $(\mathrm{EM})$ of $\sim 500 \mathrm{~cm}^{-6} \mathrm{pc}$, in reasonable agreement (given the uncertainties in this calculation) with the observed (reddening-corrected) surface brightness of $\mathrm{H} \alpha$ of $2 \times 10^{-15}$ ergs $\mathrm{s}^{-1} \mathrm{~cm}^{-2} \operatorname{arcsec}^{-2}$, corresponding to $\mathrm{EM} \approx 900 \mathrm{~cm}^{-6} \mathrm{pc}$. However, despite this nominal agreement, ionization by the AGN appears unlikely based on other line diagnostics that are independent of projection.

A line of particular interest for this purpose is He II $\lambda 4686$ since it is a gauge of the hardness of the ionizing continuum. Figure 6 shows the composite filament spectrum for NGC 1275 in the region of He II $\lambda 4686$ together with the template continuum. The observed $3 \sigma$ upper limit for He II $\lambda 4686 / \mathrm{H} \beta$ is 0.024 , after dereddening. Photoionization simulations show that this value is sensitive to the ionization parameter for a given spectral shape. Based on the continuum and nebular parameters described above, we examined this issue using photoionization calculations obtained with CLOUDY, version 90.04 (Ferland 1996). The result is a prediction that He II $\lambda 4686 / \mathrm{H} \beta \approx 0.10$, which is four times our upper limit. Therefore, the nebular filaments in NGC 1275 are highly unlikely to be powered by the AGN.

In addition to being a source of ionizing radiation, the AGN in NGC 1275 is a radio source (3C 84) with extended radio structures (Pedlar 1981 et al.; Pedlar et al. 1990), that may in principle power the optical nebulosity. The relation between the radio plasma and the nebular gas was discussed briefly by McNamara, O'Connell, \& Sarazin (1996) in a multiwavelength study dealing with NGC 1275 and its environs. The extended radio emission roughly coincides with two cavities in the X-ray gas located on the northeast and southwest outer portions of the galaxy (Pedlar et al. 1990; McNamara et al. 1996). The 
nebular emission seems to avoid the radio emission, which may be evidence for the radio lobes displacing the nebular gas. Another interpretation would be that the nebular gas, which has a higher density, may be confining the radio-emitting gas and channeling it to regions of lower density (McNamara et al. 1996). In any case, if an interaction with the radio lobes were responsible for powering the nebular emission, we might expect the optical filaments to be correlated spatially with the radio structures. This correspondence is not seen, but might indicate that the shocked gas remains in a hot phase. Recent observations with Chandra, however, show little evidence for X-ray emission by a hot shocked component in the regions in question (M. Wise, private communication); a similar situation has been reported for Hydra A (McNamara et al. 2000). As will be argued below, these results and additional spectroscopic constraints suggest that shock heating is unlikely in the filaments of NGC 1275.

\subsubsection{SHOCKS}

The above arguments point toward a distributed ionizing source as the underlying power source for the filaments in NGC 1275. This ionization mechanism could in principle be shocks, stars, and/or the cooling ICM itself. Several observational properties can be interpreted as possible evidence of shocks in the filaments. The velocity field of the nebular gas shows variations of $100-300 \mathrm{~km} \mathrm{~s}^{-1}$ (e.g., Burbidge \& Burbidge 1965; Heckman et al. 1989). Moreover, the line widths are greater than the thermal width. Line emission from gas at a temperature of $10,000 \mathrm{~K}$ will exhibit a full width at half maximum (FWHM) of $\sim 20 \mathrm{~km} \mathrm{~s}^{-1}$ for hydrogen, which can be compared with observed FWHM values of $100-300 \mathrm{~km} \mathrm{~s}^{-1}$ in the spatially-resolved spectra, after removal of the instrumental width.

Several possible mechanisms are available for driving shocks, including interaction of the radio plasma from the AGN with surrounding gas (§3.3.1), pressure imbalances resulting from thermally unstable cooling in the cluster center (Cowie, Fabian, \& Nulsen 1980), and accretion infall onto the central galaxy. For shocks driven by radio ejecta, Bicknell, Dopita, \& O'Dea (1997) have shown that the luminosity in $\mathrm{H} \alpha$ is of order $0.1 \%$ of the energy flux into the radio plasma, with the exact value dependent on the gas density distribution and shock velocity. For NGC 1275, Pedlar et al. (1990) estimate that $\sim 10^{43} \mathrm{erg} \mathrm{s}^{-1}$ is injected into the radio halo; this value is inversely related to the age of the halo, which Pedlar et al. take to be $10^{8}$ years or less. The total $\mathrm{H} \alpha$ luminosity of the NGC 1275 filaments is $\sim 2.1 \times 10^{42}$ $\operatorname{erg~s}^{-1}$ (Heckman et al. 1989; Caulet et al. 1992). It appears doubtful that the radio source is sufficient to power the observed line emission, unless the radio halo is exceedingly young or the energy transfer to optical emission is unusually efficient. 
Efficient cooling of matter near the cluster center can lead to regions that are underpressured relative to their surroundings, and hence will be subjected to repressurizing shocks (Cowie, Fabian, \& Nulsen 1980). The power delivered by such shocks results from the thermal and mechanical energy of the ICM, so that the maximum power that be can extracted from the cooling ICM is $(5 / 2)\left(k T / \mu m_{p}\right) \dot{M} \approx 10^{43} \mathrm{erg} \mathrm{s}^{-1}$. Only about $3 \%$ of this energy is processed into $\mathrm{H} \alpha$, requiring that the cooling clouds be shocked multiple times to reproduce the observed emission (the " $H_{\text {rec }}$ problem"; see Heckman et al. 1989 for a discussion). The apparent survival of grains in the filaments of other cooling-flow galaxies suggests that passage of repeated shocks through the nebular gas is unlikely (Donahue \& Voit 1993).

If the bulk kinetic energy of infalling matter dominates the energy inflow to the central region, the power available for driving shocks is of order $\dot{M} v_{i}^{2}$, where $v_{i}$ is the characteristic infall speed. If $v_{i}$ is as high as the cluster velocity dispersion $\left(\sim 1000 \mathrm{~km} \mathrm{~s}^{-1}\right.$; Kent \& Sargent 1983), the resulting rate of energy transfer is $\sim 10^{44} \mathrm{erg} \mathrm{s}^{-1}$, which is sufficient to power the filament emission. However, if $v_{i}$ is closer to the filament velocity dispersion, the available power is an order of magnitude less, and probably insufficient to generate the observed $\mathrm{H} \alpha$ luminosity.

One general characteristic of shocks is that line-intensity ratios of emitted radiation reflect the kinetic energy flux entering the shock front, and hence are a function of shock velocity (e.g., Shull \& McKee 1979; Dopita \& Sutherland 1995, Binette, Dopita, \& Tuohy 1985). If the observed line width reflects the characteristic shock velocity, then we would expect the line ratios to correlate with line width; such behavior is in fact seen in large-scale outflows from starburst galaxies (e.g., Heckman et al. 1999). A plot of $\log ([\mathrm{N} \mathrm{II}] \lambda 6583 / \mathrm{H} \alpha)$ versus the FWHM of $\mathrm{H} \alpha$ from the four PAs is shown in Figure 7, together with model predictions, which exhibit a positive trend in most cases. The range of observed FWHM is $100-300 \mathrm{~km} \mathrm{~s}^{-1}$, while $[\mathrm{N} \mathrm{II}] / \mathrm{H} \alpha$ is clustered around 0.9 . A shallow but statistically significant correlation (Spearman non-parametric significance of $\sim 5 \times 10^{-3}$ ) is present, with larger line ratios associated with higher velocity width. This trend is due to combining values from all the $\mathrm{PAs} ; \mathrm{PA}=283^{\circ}$ and $\mathrm{PA}=313^{\circ}$ have slightly higher line ratios and $\mathrm{FWHM}$ than $\mathrm{PA}=62^{\circ}$ and $\mathrm{PA}=77^{\circ}$. Significant correlations are not seen in either the individual PAs or in PA62/PA77 and PA283/PA313 groupings, making shocks as heating sources in the filaments unlikely. Similar behavior is seen in the $[\mathrm{O} \mathrm{III}] / \mathrm{H} \beta$ ratios, although no correlation or PA-dependent behavior is evident in $[\mathrm{S} \mathrm{II}] / \mathrm{H} \alpha$ or $[\mathrm{O} \mathrm{I}] / \mathrm{H} \alpha$.

A further piece of evidence which argues against shocks comes from the upper limit on electron temperature as determined from the [O III] lines (Table 1). Figure 8 shows the continuum around the expected location of $[\mathrm{O}$ III $] \lambda 4363$. An upper limit on the temperature as measured from $[\mathrm{O}$ III $] \lambda 4363 /[\mathrm{O}$ III $] \lambda \lambda 4959,5007<0.047$ restricts the electron 
temperature to be less than 33,000 K (Osterbrock 1989; Shaw \& Dufour 1995). Shock models predict [O III] $\lambda 4363 /[\mathrm{O}$ III $] \lambda \lambda 4959,5007$ to be between 0.01 and 0.07 (Shull \& McKee 1979; Raymond 1979; Binette et al. 1985; Dopita \& Sutherland 1995), corresponding to electron temperatures between 13,000 K and 54,000 K. Shock models with velocities less than $\sim 300 \mathrm{~km} \mathrm{~s}^{-1}$ predict an [O III] line ratio consistent with our upper limit, but have problems in simultaneously matching the high- and low-ionization forbidden-line strengths. These models also consistently underpredict He I $\lambda 5876$, except at high velocities, where the predicted He II $\lambda 4686$ exceeds our upper limit (Raymond 1979; Binette et al. 1985; Dopita \& Sutherland 1995). Therefore, we conclude that shock heating in the filaments of NGC 1275 is a minor contributor to the energetics of the nebular gas.

\subsubsection{Stellar Photoionization}

Cooling-flow galaxies commonly exhibit an optical continuum that is bluer than typical elliptical galaxies (e.g., Johnstone, Fabian, \& Nulsen 1987; McNamara \& O'Connell 1989; Allen 1995; Cardiel, Gorgas, \& Aragon-Salamanca 1995, 1998). Some degree of correlation exists between such blue continua and nebular emission, prompting suggestions that both may trace the influence of an underlying young stellar population. While nebular emission (bremsstrahlung, free-bound, and 2-photon) may contribute to such a continuum, a quantitative calculation indicates that the continuum emission accompanying the observed $\mathrm{H} \beta$ luminosity would be 2 orders of magnitude too weak to account for the observed light. Stars are thus the likely source of the blue continua. As possible ionizing agents for the nebular gas, stars also have the added appeal of producing negligible He II nebular emission, consistent with the observations of NGC 1275 and other cooling-flow galaxies.

Early studies of the large-scale nebular morphology of NGC 1275 interpreted this object and its system of radial filaments as the result of a large-scale outflow; indeed, it was called an "exploding galaxy" (Burbidge \& Burbidge 1965). Modern studies of another object given this label, M82, demonstrate that it is in a sense "exploding" because of its large-scale outflow driven by star formation (e.g., McCarthy et al. 1987; Shopbell \& Bland-Hawthorn 1998). However, such vigorous star formation is not apparent in NGC 1275.

NGC 1275 does show weaker indications of star formation within the last $\sim 10^{7}-10^{8}$ yrs on a variety of scales (Wirth et al. 1983; Holtzman et al. 1992; McNamara et al. 1996). Optical measures of the young population have been used to estimate roughly the associated ionizing luminosity, yielding a result that is consistent with the requirements of powering the filaments (Wirth et al. 1983; Romanishin 1987). However, there is not a strong spatial

correspondence between the regions of recent star formation and the nebular gas. As noted 
previously, the nebular spectrum also differs markedly from typical H II region spectra; in the one instance where recent star formation is clearly evident within a filament (at PA = $62^{\circ}$ ), the nebular line ratios are distinct from the remainder of the filament system (Shields \& Filippenko 1990a). Photoionization models suggest that hot stars can generate LINERlike emission (Filippenko \& Terlevich 1992; Shields 1992), but these scenarios generally have difficulty producing $[\mathrm{O} \mathrm{I}]$ and $[\mathrm{N} \mathrm{I}]$ emission as strong as seen here, unless the gas contains a very high density component $\left(\sim 10^{5} \mathrm{~cm}^{-3}\right)$.

Stars may nonetheless play a role in powering the filaments if they generate a radiation field that ionizes gas on scales exceeding that of localized H II regions. A similar scenario has recently gained favor for explaining the ionization of the diffuse ionized gas (DIG) commonly seen in spiral galaxies (e.g., Howk \& Sembach 1999; Rand 1998; Martin 1997). However, the forbidden-line strengths seen in the DIG are characteristically weaker than in the NGC 1275 filaments; typical DIG line ratios are $[\mathrm{O} \mathrm{I}] \lambda 6300 / \mathrm{H} \alpha \lesssim 0.03,[\mathrm{~N}$ II $] \lambda 6583 / \mathrm{H} \alpha \approx 0.5$, He I $\lambda 5876 / \mathrm{H} \alpha \lesssim 0.02$, and [O III] $\lambda 5007 / \mathrm{H} \alpha \lesssim 0.1$ (Galarza, Walterbos, \& Braun 1999; Sembach et al. 2000), which contrasts markedly with the values in Table 1. Another aspect which makes the cooling-flow filaments of NGC 1275 distinct from the DIG is the emission measure. For the DIG, EM $\approx 1-10 \mathrm{~cm}^{-6}$ pc (Reynolds 1985; Martin \& Kennicutt 1997; Hoopes, Walterbos, \& Rand 1999), and can reach values as high as $100 \mathrm{~cm}^{-6}$ pc (Galarza et al. 1999; Hoopes et al. 1999). In contrast, the average EM in NGC 1275 for the regions studied here is $\sim 900 \mathrm{~cm}^{-6} \mathrm{pc}$.

Given the arguments for and against stellar photoionization, it may be the case that this excitation process, if present, is at work in combination with another ionization source, as has been pointed out by Voit \& Donahue (1997) for Abell 2597. The evidence from NGC 1275, however, does not form a strong case for stellar photoionization as the dominant heating mechanism in the filaments.

\subsubsection{Photoionization By the ICM}

The hot ICM surrounding NGC 1275 can be the ionization source of the filaments if its energy is channeled efficiently into the nebulosity (Voit \& Donahue 1990; Begelman \& Fabian 1990). In one such scenario, known as self-irradiation, the hot ICM ionizes nebulae that have already cooled and condensed from the ICM itself (Donahue \& Voit 1991; Voit \& Donahue 1990; Voit et al. 1994). Another way to channel ICM energy into the nebulae is the formation of mixing layers between cold clouds $\left(T_{e} \approx 10^{4} \mathrm{~K}\right)$ embedded in a hot ambient medium $\left(T_{e} \approx 10^{7} \mathrm{~K}\right)$ (Begelman \& Fabian 1990; Crawford \& Fabian 1992). Line emission comes from the mixing layer, which is described by an intermediate temperature $\left(T_{e} \approx 10^{5.5}\right.$ 
$\mathrm{K}$ ), and from the cool cloud that is now being irradiated by the mixing layer. Crawford \& Fabian (1992) were able to reproduce the line ratios of Abell 496, a cluster that displays Type I filaments, with a mixing-layer prescription. However, in order to reproduce the line ratios of the Type II cluster Abell 2597, these authors found it necessary to invoke an additional energetic contribution from shocks.

The observed line ratios in NGC 1275 are close to values predicted by one of the selfirradiation models presented by Voit et al. (1994). In that model (D39T67), the ICM is cooling from a temperature of $5 \times 10^{6} \mathrm{~K}$, the ionization parameter is $10^{-3.9}$, and the column density of irradiated H I is $10^{21.5} \mathrm{~cm}^{-2}$. Their predicted He II $\lambda 4686 / \mathrm{H} \beta$ ratio is almost a factor of 3 larger than our upper limit. This comparison shows that self-irradiation is not the ionization mechanism because it provides a continuum which is harder than that inferred from the observed line ratios. A cooling-flow system that shows similar filaments, in terms of line ratios, to NGC 1275 is Abell 2597 (Heckman et al. 1989; Voit \& Donahue 1997). Voit \& Donahue (1997) similarly ruled out a hard ionizing continuum as the energy source in the filaments of Abell 2597 on the basis of an upper limit of 0.02 on He II $\lambda 4686 / \mathrm{H} \beta$, which is again a factor of $\sim 3$ below expectations for self-irradiation.

\subsubsection{Composite Ionization Models}

None of the ionization mechanisms considered individually in the preceding sections is entirely satisfactory in accounting for the emission properties and energetics of the coolingflow filaments in NGC 1275. Given the compelling evidence for the presence of young stars, it is reasonable to explore models that are composites of stellar photoionization and some secondary heating mechanism. The study by Voit \& Donahue (1997) of Abell 2597 is illuminating in this regard. Voit \& Donahue (1997) invoked stellar photoionization as the ionizing mechanism for the optical filaments of Abell 2597 after placing strong upper limits on [O III] $\lambda 4363$ and He II $\lambda 4686$, a result that ruled out shock heating and selfirradiation by the ICM. However, they found that the electron temperature predicted for stellar photoionization was lower than what they inferred from their observations of the [O II] and [S II] lines. For this reason, they suggested that a heating source in addition to hot stars may be important. The secondary source they considered was the ICM; it can heat the filaments via electron conduction (e.g., Sparks 1992), acoustic-wave heating (e.g., Pringle 1989), and MHD-wave heating (e.g., Friaca et al. 1997). Voit \& Donahue (1997) argued that the resulting energy flux incident on the nebulae can become greater than that of photoelectric heating if the nebulae are characterized by low ionization, a case which

holds in cooling-flow filaments. Unfortunately, detailed theoretical predictions appropriate 
for comparison with observations have not yet been generated for these scenarios. A related case where the heating rates due to the dissipation of turbulence have been calculated and found to be comparable to the radiative cooling rates is in the DIG of the Milky Way (Minter \& Spangler 1997). However, detailed observational parameters (such as the amplitude of density fluctuations, and turbulence length scales) needed to calculate the heating rate from this mechanism are unavailable for cooling-flow filaments.

We investigated an alternative scenario in which the observed emission arises from a combination of clouds subject to stellar photoionization and irradiation by the ICM. In this simplified model, we assumed that within a given measurement aperture, we see a mix of clouds ionized by stars and by radiation from the ICM. In this picture we observe a weighted average of the spectrum of the two types of cloud. A fraction of the observed line emission comes from regions ionized by stars, while another fraction comes from nebulae irradiated by the ICM. This weighted average translates into the corresponding fractions in terms of line ratios (with all ratios normalized to $\mathrm{H} \beta$ ). We used line ratios from the self-irradiation models of Voit et al. (1994), and representative values for intermediate-metallicity H II regions (Veilleux \& Osterbrock 1987). Once the relative contributions of stars and the ICM were determined from He II $\lambda 4686 / \mathrm{H} \beta$, attention was turned to reproducing [O I] $\lambda 6300 / \mathrm{H} \alpha$ as well as the other line ratios. [O I] $\lambda 6300 / \mathrm{H} \alpha$ is a good diagnostic because it is very weak in $\mathrm{H}$ II regions. Including more stars pushed $[\mathrm{O} \mathrm{I}] \lambda 6300 / \mathrm{H} \alpha$ below observed values, while more self-irradiation would push He II $\lambda 4686 / \mathrm{H} \beta$ above what is observed. Ultimately we were not able to find a composite model that fits all the line ratios simultaneously. Combinations satisfying the helium requirement, with marginal agreement for the other line ratios, failed to match the oxygen constraint, and vice versa. Composite models can be found that satisfy both $[\mathrm{O} \mathrm{I}] / \mathrm{H} \alpha$ and $\mathrm{He} \mathrm{II} / \mathrm{H} \beta$, but these scenarios proved inconsistent with the other line ratios. The reason for this failure to come up with a successful hybrid model may be due to our simplistic approach, which neglects clouds that see ionizing radiation from both sources simultaneously, or more likely suggests that other ionization mechanisms are at work.

\subsubsection{Attenuated Cluster Radiation}

If the filaments in NGC 1275 and other cooling-flow galaxies are photoionized, hints about the nature of the ionizing continuum come from the strengths of the emission lines. Relatively strong forbidden lines, the absence of He II $\lambda 4686$, and the presence of He I $\lambda 5876$ suggest that the ionizing continuum cuts off at energies somewhere between 24 and $54 \mathrm{eV}$. An ionizing source generating a continuum with a natural cutoff at $54 \mathrm{eV}$ is normal O-type stars. For this reason we tried in the previous subsection to construct a composite model of 
stellar photoionization and self-irradiation by the ICM, but our attempts were unsuccessful in terms of matching the observed line ratios.

The cooling ICM can produce a range of ionizing continua, depending on its initial temperature. Self-irradiation models (Voit et al. 1994) that predict a He II $\lambda 4686$ value similar to what is observed fail at reproducing the other line ratios, while self-irradiation models that agree with most of the observed line ratios have high He II $\lambda 4686 / \mathrm{H} \beta$. One way to construct an appropriate ionizing spectrum with the shape dictated by the observations is to introduce a cutoff at the helium edge of $54 \mathrm{eV}$ by attenuating the spectrum by an ionized screen. The filtering medium will imprint a sharp dip at $54 \mathrm{eV}$ while leaving the flux of photons with energies between 24 and $54 \mathrm{eV}$ nearly unchanged. Observational evidence for such a screen may come from X-ray observations of Abell 426 that suggest the presence of absorbing matter with a column density of $N_{H} \approx 10^{21} \mathrm{~cm}^{-2}$, in excess of the Galactic foreground component; the ionization state of this medium is not well understood (e.g., Allen, Fabian, \& Edge 1992; Arnaud \& Mushotzky 1998).

We therefore constructed models in which the ionizing continuum from the cooling ICM is attenuated through a screen before irradiating the filaments. This scenario differs from the traditional self-irradiation models of Voit \& Donahue (1990) in the sense that the filaments and the screen need not be the by-products of the cooling flow. The models discussed here also differ from the mixing layers picture of Crawford \& Fabian (1992) in terms of the origin of the ionizing continuum. In the mixing layers model, the ionizing spectrum comes from gas at a temperature intermediate between the temperature of the ICM and that of the optical filaments.

To test the photoionization plus screen scenario, we carried out photoionization calculations using CLOUDY, version 90.04 (Ferland 1996). We assumed plane-parallel geometry throughout the calculations, and a metallicity of 0.4 times solar values, consistent with X-ray studies (e.g., Allen et al. 1992). The flux originating from the ICM was scaled to match the soft X-ray central surface brightness reported by Mohr, Mathiesen, \& Evrard (1999), and propagated consistently through the calculation. We found good results assuming a temperature for the dominant ionizing plasma of $10^{6} \mathrm{~K}$. While lower than that of the majority of the ICM, this value is in general accord with the high degree of cooling occurring in the Perseus cluster core, and with spectroscopic evidence for cooler phases (e.g., Mushotzky \& Szymkowiak 1988) in this region.

In order to have the appropriate screening properties, the ionization state of the filtering medium should be such that the optical depth at $54 \mathrm{eV}$ is large enough to suppress He II $\lambda 4686$ in the filaments, while the total hydrogen column density stays less than, or comparable to, the column density inferred from X-ray observations. Moreover, the ionization 
parameter of the screen should be high enough to produce minimal optical forbidden-line emission, since the screen is evidently not seen in such lines. Therefore, we chose the hydrogen density in the screen to be $1 \mathrm{~cm}^{-3}$, implying an ionization parameter of $U \approx 10^{-1.1}$, and stopped the calculation when the optical depth at $54 \mathrm{eV}$ became 10 . The resulting hydrogen column density in the screen is $\sim 6 \times 10^{21} \mathrm{~cm}^{-2}$. For the filament, the forbidden-line spectrum suggests an ionization parameter $U \approx 10^{-3.9}$ (§3.3.1). This value emerges naturally from the propagated radiation field and a choice of density of $\sim 100 \mathrm{~cm}^{-3}$, consistent with the observed $[\mathrm{S}$ II] $\lambda 6717 / \lambda 6731$ line ratio.

Resulting predictions for the emission-line spectrum emergent from a filament are shown in Table 3. All the line ratios were satisfactorily reproduced, with the exception of the [S II] lines, which are too strong by a factor of $\sim 1.6$. Figure 9 shows the continuum incident on the screen, and the continuum exiting the screen and incident on the filament. Notice the strong ionized helium edge at $54 \mathrm{eV}$. Since the ionized screen is photoionized, it will also emit recombination radiation, especially in He II $\lambda 4686$ (He II Pa $\alpha$ ). In a sense, then, this model removes the difficulty of excess He II emission in the filaments, but probably creates a new observational inconsistency in that the screen should be a strong source of observable recombination emission, which is not apparently seen.

He II $\lambda 4686$ emission would be reduced by a factor of $\sim 2$ if recombination in the screen were described by Case A conditions rather than Case B. However, the line center optical depth for He II Ly $\gamma$, which upon reabsorption can branch to emit the Pa $\alpha$ line, remains $\gg 1$ for the column density and temperature describing our model screen; additional turbulence of $\sim 10^{4}-10^{5} \mathrm{~km} \mathrm{~s}^{-1}$ within the screen would be required to yield Case A conditions. Case A recombination thus does not appear to be a plausible mechanism for reducing emission in He II $\lambda 4686$.

\subsubsection{Diffuse Extreme Ultraviolet Radiation}

Observations with the Extreme Ultraviolet Explorer (EUVE) indicate the presence of excess soft X-ray/EUV emission in clusters of galaxies (Lieu et al. 1996; Lieu, Bonamente, \& Mittaz 1999). The existence and properties of this emission are still highly debated (e.g., Arabadjis \& Bregman 1999). If this emission component is real, an interesting question is whether it plays a significant role in the energetics of the nebular filaments.

A difficulty with using the EUV observations directly stems from the fact that EUVE data (Lieu et al. 1996) cover only a limited bandpass, making any attempts at determining the total ionizing luminosity dependent upon the shape of the assumed continuum, a property 
that has to be determined by a model for the origin of the EUV excess. According to one interpretation, inverse-Compton scattering of the cosmic microwave background radiation off cosmic-ray electrons is responsible for the EUV continuum, which is then described by a two-point spectral index between 70 and $912 \AA$ of $\alpha_{E U V}=-2 / 3$ (Sarazin \& Lieu 1998; Sarazin 1999). With this spectral energy distribution, the ratio of photon surface brightness between $70 \AA$ and $912 \AA$ to that between $70 \AA$ and $160 \AA$, the approximate range covered by the EUVE DS Lexan detector, is about 6.2 .

No EUVE observations have been made of NGC 1275; the large Galactic H I column density toward this source $\left(1.4 \times 10^{21} \mathrm{~cm}^{-2}\right.$; Lockman \& Savage 1995) makes its detection difficult or impossible at EUV wavelengths. In order to obtain an order-of-magnitude estimate of the relevant photon flux in the EUVE bandpass, we used observations of Abell 1795 (Lieu et al. 1999). Abell 1795 is a cooling-flow cluster whose central galaxy displays bright optical filaments which, like those in NGC 1275, were classified spectroscopically by Heckman et al. (1989) as Type II; the $\dot{M}$ values inferred from X-ray observations agree to within a factor of $\sim 2$ for the two clusters. Assuming an effective area of $\sim 15 \mathrm{~cm}^{2}$ for EUVE with the DS Lexan detector (Miller 1997), the central surface brightness for Abell 1795 is $2.0 \times 10^{-4}$ photons $\mathrm{s}^{-1} \mathrm{~cm}^{-2} \operatorname{arcmin}^{-2}$ (Lieu et al. 1999). Scaling this value to obtain the ionizing $(70 \AA$ - $912 \AA$ ) surface brightness results in a value of $\sim 1.2 \times 10^{-3}$ photons s${ }^{-1} \mathrm{~cm}^{-2} \operatorname{arcmin}^{-2}$. If we assume that the filaments can be treated as ionization-bounded slabs located at the center of the EUV-emitting medium, the ionizing photon flux striking the optical gas is $\sim 4.5 \times 10^{4}$ photons $\mathrm{s}^{-1} \mathrm{~cm}^{-2}$, implying $\mathrm{EM} \approx 0.06 \mathrm{~cm}^{-6} \mathrm{pc}$ in the photoionized slab. This value differs substantially from the emission measure calculated above from our $\mathrm{H} \alpha$ surface brightness for NGC 1275, $900 \mathrm{~cm}^{-6} \mathrm{pc}$, which is also comparable to the results obtained for other systems (Voit \& Donahue 1997). We conclude that the EUV emission, if present in Abell 426, is a highly unlikely ionizing mechanism for the optical filaments in NGC 1275.

\subsubsection{MAgnetic ReCOnNECTION}

Another source of energy in galaxy clusters is the intergalactic magnetic field. Magnetic reconnection of field lines in clusters can release energy in a manner similar to that of solar flares (e.g., Heckman et al. 1989; Soker \& Sarazin 1990). For magnetic reconnection to be effective in releasing energy, the magnetic field must be strong and tangled. Reconnection of magnetic flux loops will liberate energy which has to be included in the energy budget of the cooling-flow filaments (e.g. Soker \& Sarazin 1990; Jafelice \& Friaca 1996).

Cooling flows present in galaxy clusters may amplify the magnetic field energy density

to equipartition values with the gas pressure within the inner tens of kpc due to compression 
of the field lines that are frozen-in to the plasma (e.g., Soker \& Sarazin 1990). It is interesting to note that the observed optical filaments in cooling-flow galaxies occur over the same length scale predicted for equipartition between magnetic and gas pressures, for theoretical coolingflow models. The resulting strong magnetic fields are expected to produce large Faraday rotations in the cores of cooling-flow clusters, in agreement with radio measurements for many sources (Ge \& Owen 1993, 1994). As a result of this amplification, one would expect clusters with larger cooling rates to have larger Faraday rotation measures.

The correlation between cooling rates and Faraday rotation measure seems to be borne out by observations. Taylor, Barton, \& Ge (1994) compiled a list of radio sources at the centers of galaxy clusters, and showed that such a trend exists. Notable exceptions, however, are NGC 1275 and PKS 0745-191, which have strong cooling flows and extended optical nebulae, but no measurable polarization (Ge \& Roberts 1993; Taylor et al. 1994). One possible interpretation of this finding is that a magnetized screen with complex structure is strongly depolarizing the radio signal (Ge \& Roberts 1993; Taylor et al. 1994). The screen may be the optical filaments themselves. Several pieces of observational evidence indicate that this might be the case. The $\mathrm{H} \alpha$ and radio emission are cospatial in projection in PKS 0745-191 and in the core of NGC 1275 (Heckman et al. 1989; Ge \& Roberts 1993). Van Breugel, Heckman, \& Miley (1984) noted that there is a spatial inverse correlation between radio polarization and $\mathrm{H} \alpha$ surface brightness in Abell 1795, a result later confirmed by Ge \& Owen (1993) at higher spatial resolution. Obtaining more radio polarimetry data with high spatial resolution for cooling-flow clusters will be very helpful in testing this inverse correlation. A detailed rotation measure map will make it possible to look for a relation between the $\mathrm{H} \alpha$ luminosity of a filament and the degree of depolarization it imprints on the radio emission cospatial with it.

The above discussion provides circumstantial evidence for the importance of magnetic fields in understanding cooling-flow filaments. If Faraday depolarization in the optical filaments can be taken as evidence for the filaments being magnetized with a complex geometry, then magnetic field lines may be reconnecting in the nebular gas, and one would expect strong depolarization to be associated with strong optical emission. In terms of energetics, Jafelice \& Friaca (1996) argued, on the basis of theoretical models, that magnetic reconnection is capable of powering the lower luminosity Type I filaments, but falls short of sustaining the Type II systems, such as NGC 1275, Abell 1795, and PKS 0745-191. Additional high spatial resolution radio polarimetry for cooling-flow galaxies is desirable in order to carry out a comparative study of the magnetic properties of the two source types. A further interesting experiment would be to obtain such data for the central galaxy in Sérsic 159-03 (S 1101), which displays filaments with emission-line ratios encompassing both Type I and Type II values (Crawford \& Fabian 1992). 


\section{Conclusions}

In this paper we have presented the results of a detailed optical spectroscopic study of the optical filaments around NGC 1275, the central galaxy in the Perseus cluster of galaxies. The database was extensive enough to yield tight upper limits on weak lines of interest for constraining the ionization and thermal state of the nebular gas. Line-ratio diagrams reveal that the nebulosity has an unusual combination of emission-line strengths reflecting both low ionization and low excitation.

The spectroscopic results were used to gauge the viability of several scenarios proposed for powering cooling-flow nebulosity. Mechanisms examined include shocks, ionization by an AGN central source or extended radio structures, stellar photoionization, and energy transfer by various means from the ICM. None of these models is entirely satisfactory in accounting for the energetic and/or emission-line properties of the filaments. Radio studies of Faraday rotation for cooling-flow galaxies provide tantalizing suggestions that magnetic reconnection plays a significant role in the filament energetics. Additional polarization studies of these sources may provide a clearer picture as to the importance of magnetic fields in these systems.

Support for this work was provided in part by a grant from the California Space Institute to J.C.S., by NSF grants AST-8957063 and AST-9417213 to A.V.F., and by NASA grant NAG5-3556 to A.V.F. We thank the Mount Hamilton staff of Lick Observatory for their friendly and capable assistance over the multiple observing runs required for this project. At the time when the observations were obtained, Lick Observatory received partial funding from the NSF through Core Block Grant AST-8614510 to the University of California. We also thank Ray Bertram and Rick Pogge for assistance at the Perkins 1.8-m telescope. We

gratefully acknowledge Gary Ferland for making CLOUDY available for this study, and Mike Wise for communicating results from Chandra observations in advance of publication.

\section{REFERENCES}

Arabadjis, J. S., \& Bregman, J. N. 1999, ApJ, 514, 607

Allen, S. W. 1995, MNRAS, 276, 947

Allen, S. W., Fabian, A. C., Johnstone, R. M., Arnaud, K. E., \& Nulsen, P. E. J. 2000, MNRAS, submitted (astro-ph/9910188)

Allen, S. W., Fabian, A. C., Nulsen, P. E. J., \& Edge, A.C. 1992, MNRAS, 254, 51

Arnaud, K. A., \& Mushotzky, R. F. 1998, ApJ, 501, 119 
Baldwin, J. A., Phillips, M. M., \& Terlevich, R. 1981, PASP, 93, 5

Begelman, M. C., \& Fabian, A. C. 1990, MNRAS, 244,26P

Bicknell, G. V., Dopita, M. A., \& O’Dea, C. P. O. 1997, ApJ, 485, 112

Binette, L., Dopita, M. A., \& Tuohy, I. R. 1985, ApJ, 297, 476

Branduardi-Raymont, G., Fabricant, D., Feigelson, E., Gorenstein, J., Grindlay, J., Soltan, A., \& Zamorani, G. 1981, ApJ, 248, 55

Burbidge, E. M., \& Burbidge, G. R. 1965, ApJ, 142, 1351

Burstein, D., \& Heiles, C. 1984, ApJS, 54, 33

Cardelli, J. A., Clayton, G. C., \& Mathis, J. S. 1989, ApJ, 345, 245

Cardiel, N., Gorgas, J., \& Aragon-Salamanca, A. 1995, MNRAS, 277, 502

Cardiel, N., Gorgas, J., \& Aragon-Salamanca, A. 1998, MNRAS, 298, 977

Caulet, A., et al. 1992, ApJ, 388, 301

Cowie, L. L., Fabian, A. C., \& Nulsen, P. E. J. 1980, MNRAS, 191, 399

Crawford, C., \& Fabian, A. C. 1992, MNRAS, 259, 265

Donahue, M., \& Stocke, J. T. 1994, ApJ, 422, 459

Donahue, M., \& Voit, G. M. 1991, ApJ, 381, 361

Donahue, M., \& Voit, G. M. 1993, ApJ, 414, L17

Dopita, M. A., \& Sutherland, R. S. 1995, ApJS, 102, 161

Fabian, A. C., Cowie, L. L., \& Grindlay, J. 1981, ApJ, 248, 47

Fabian, A. C., Nulsen, P. E. J., \& Canizares, C. R. 1984, Nature, 310, 733

Ferguson, J. W., Korista, K. T., \& Ferland, G. 1997, ApJS, 110, 287

Ferland, G. J. 1996, Hazy, a Brief Introduction to CLOUDY, University of Kentucky Department of Physics and Astronomy Internal Report

Ferland, G. J., \& Netzer, H. 1983, ApJ, 264, 105

Filippenko, A. V., \& W. L. W. Sargent 1985, ApJS, 85, 503

Filippenko, A. V., \& Terlevich, R. 1992, ApJ, 397, L79

Friaca, A. C. E., Gonvalves, D. R., Jafelice, L. C., Jatenco-Pereira, V., \& Opher, R. 1997, MNRAS, 324, 449

Galarza, V. C., Walterbos, R. A. M., \& Braun, R. 1999, AJ, 118, 2775

Ge, J. P., \& Owen, F. N. 1993, AJ, 105, 778 
Ge, J. P., \& Roberts, J. D. 1993, BAAS, 183, 53.10

Heckman, T. M. 1980, A\&A, 87, 152

Heckman, T. M., Armus, L., Weaver, K. A., \& Wang, J. 1999, ApJ, 517, 130

Heckman, T. M., Baum, S. A., van Breugel, W. J. M., \& McCarthy, P. J. 1989, ApJ, 338, 48

Held, E. V., Mould, J. R., \& de Zeeuw, P. T. 1990, AJ, 100, 415

Holtzman, E. J., et al. 1992, AJ, 103, 691

Hoopes, C. G., Walterbos, R. A. M., \& Rand, R. 1999, ApJ, 522, 669

Howk, J. C., \& Sembach, K. R. 1999, ApJ, 523, L141

Hu, M. E., Cowie, L. L., \& Wang, Z. 1985, ApJS, 59, 447

Hummer, D. G., \& Storey P. J. 1987, MNRAS, 224, 801

Jafelice, L. C., \& Friace, A. C. S. 1996, MNRAS, 280, 438

Johnstone, R. M., \& Fabian, A. C. 1988, MNRAS, 233, 581

Johnstone, R. M., Fabian, A. C., \& Nulsen, P. E. J. 1987, MNRAS, 224, 75

Kent, S. M., \& Sargent, W. L. W. 1979, ApJ, 230, 667

Kent, S. M., \& Sargent, W. L. W. 1983, AJ, 88, 697

Kinney, A. L., Bohlin, R. C., Blades, J. C., \& York, D. G. 1991, ApJS, 75, 645

Kriss, G. 1994, in ASP Conf. Ser. 61, Astronomical Data Analysis Software and Systems III, ed. D. R. Crabtree, R. J. Hanisch, \& J. Barnes (San Francisco: ASP), 437

Lieu, R., Bonamente, M., \& Mittaz, J. P. D. 1999, ApJ, 517, L91

Lieu, R., Mittaz, J. P. D., Bowyer, S., Lockman, F., \& Hwang, C. 1996, ApJ, 458, L5

Lockman, F. J., \& Savage, B. D. 1995, ApJS, 97, 1

Martin, C. L. 1997, ApJ, 491, 561

Martin, C. L., \& Kennicutt, R. C. Jr. 1997, ApJ, 483, 698

McNamara, B. R., \& O’Connell, R. W. 1989, AJ, 98, 2018

McNamara, B. R., O’Connell, R. W., \& Sarazin, C. L. 1996, AJ, 112, 91

McNamara, B. R., et al. 2000, ApJ, 534, L135

Miller, A. 1997, EUVE Guest Observer Program Handbook, 4th Ed. (Berkeley, CA: EGO Center) 
Miller, J. S., \& Stone, R. P. S. 1987, Lick Observatory Technical Report No. 48, The CCD Cassegrain Spectrograph at the Shane Reflector (Santa Cruz: Lick Observatory)

Minkowski, B. R. 1957, in IAU Symp. 4, Radio Astronomy, ed. H. C. van der Hulst (Cambridge: Cambridge Univ. Press), 107

Minter, T., \& Spangler, S. R. 1997, ApJ, 485, 182

Mohan, M., Hibbert, A., \& Kingston, A. E. 1994, ApJ, 434, 389

Mohr, J. J., Mathiesen, B., \& Evrard, A. E. 1999, ApJ, 517

Mushotzky, R. F., \& Szymokowiak, A. E. 1988, in Cooling Flows in Clusters and Galaxies, ed. A. C. Fabian, (Dordrecht: Kluwer), 53

Nelson, C. H., \& Whittle, M. 1995, ApJS, 99, 67

Oliva, E. 1997, in ASP Conf. Ser. 113, Emission Lines in Active Galaxies: New Methods and Techniques, ed. B. M. Peterson, F. Cheng, \& A. S. Wilson (San Francisco: ASP), 228

Osterbrock, D. E. 1989, Astrophysics of Gaseous Nebulae and Active Galactic Nuclei (Mill Valley, CA: University Science Books)

Pedlar, A., Booler, V., \& Davies, R. V. 1983, MNRAS, 203, 667

Pedlar, A., Ghantaure, H. S., Davies, R. V., Harrison, B. A., Perley, R., Crane, P. C., \& Unger, S. W. 1990, MNRAS, 246, 477

Pelan, J., \& Berrington, K. A. 1995, A\&A, 110, 209

Pringle, J. E. 1989, MNRAS, 239, 479

Rand, R. 1998, ApJ, 501, 137

Raymond, J. C. 1979, ApJS, 39, 1

Reynolds, R. J. 1985, ApJ, 294, 256

Romanishin, W. 1987, ApJ, 323, L113

Rubin, V. C., Ford, W. K., Peterson, C. J., \& Lynds, C. R. 1978, ApJS, 37, 235

Sarazin, C. L. 1999, ApJ, 520, 529

Sarazin, C. L., \& Lieu, R. 1998, ApJ, 494, L177

Sarazin, C. L., \& Graney, C. M. 1991, ApJ, 375, 532

Sembach, K. R., Howk, J. C., Ryans, R. S. I., \& Keenan, F. P. 2000, ApJ, 528, 310

Seyfert, C. A., 1943, ApJ, 97, 28

Shaw, R. A., \& Dufour, R. J. 1995, PASP, 107, 896 
Shields, J. C., 1992, ApJ, 399, L27

Shields, J. C., \& Filippenko, A. V. 1990a, ApJ, 353, L7

Shields, J. C., \& Filippenko, A. V. 1990b, AJ, 100, 1034

Shopbell, P. L., \& Bland-Hawthorn, J. 1998, ApJ, 493, 129

Shull, J. M., \& McKee, C. 1979, ApJ, 227, 131

Sparks, W. 1992, ApJ, 399, 66

Storey, P. J., Mason, H. E., \& Saraph, H. E., 1996, A\&A, 309, 677

Soker, N., \& Sarazin, C. L. 1990, ApJ, 348, 73

Taylor, G. B., Barton, E. J., \& Ge, J. P. 1994, AJ, 107, 1942

Unger, S. W., Taylor, K., Pedlar, A., Ghantaure, H. S., Penston, M. V., \& Robinson, A. 1990, MNRAS, 242, 33

van Breugel, W., Heckman, T. M., \& Miley, G. 1984, ApJ, 276, 79

Veilleux, S., \& Osterbrock, D. E. 1987, ApJS, 63, 295

Voit, G. M., \& Donahue, M. 1997, ApJ, 486, 242

Voit, G. M., \& Donahue, M. 1990, ApJ, 360, L15

Voit, G. M., \& Donahue, M., \& Slavin, J. D. 1994, ApJS, 95, 87

Wirth, A., Kenyon, S. J., \& Hunter, D. A. 1983, ApJ, 269, 102

Yan, L., \& Cohen, J. G. 1995, ApJ, 454, 44 


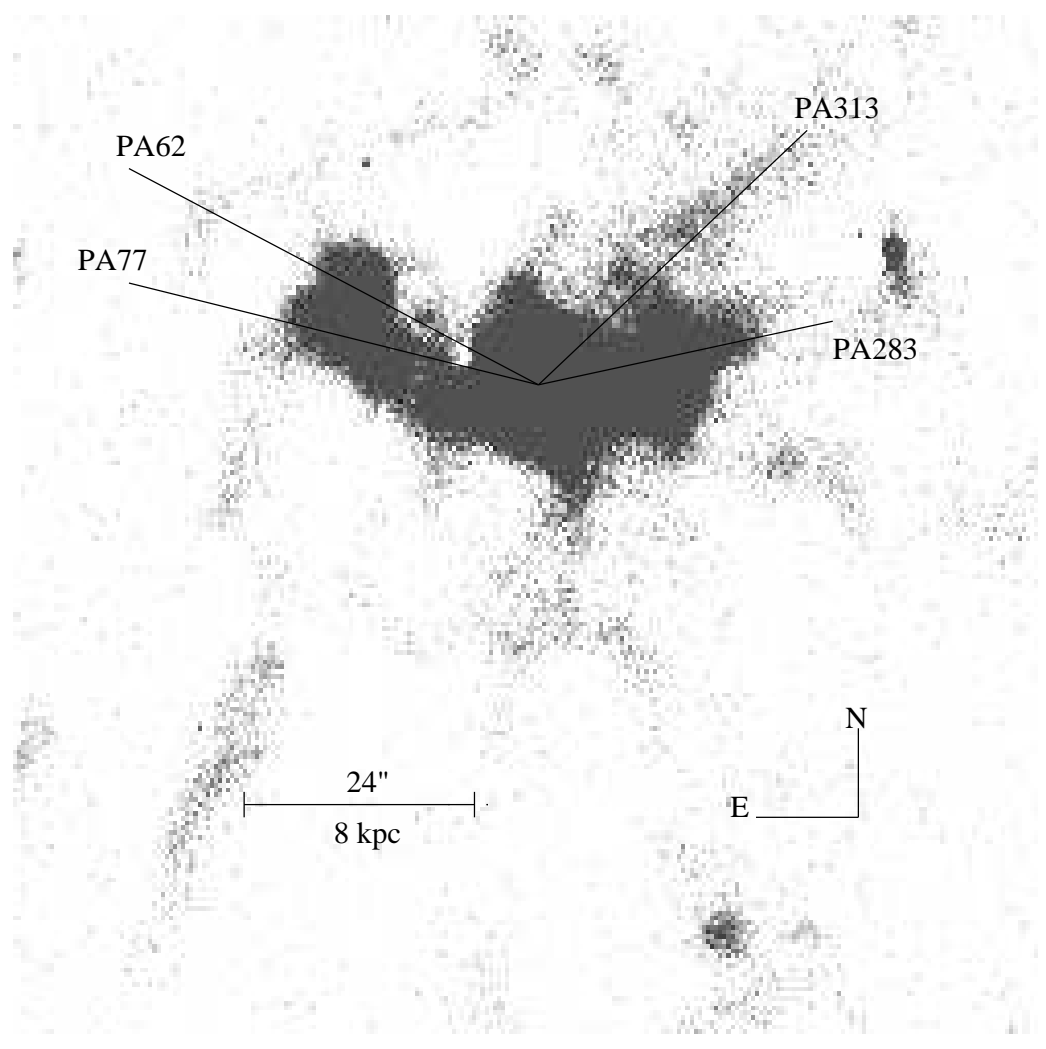

Fig. 1.- Continuum-subtracted H $\alpha+[\mathrm{N}$ II] image of NGC 1275 obtained on 1992 January 2 U.T. by Shields and R. Pogge at the Perkins 1.8-m telescope, showing the position angles of the slit employed for the measurements presented here. 


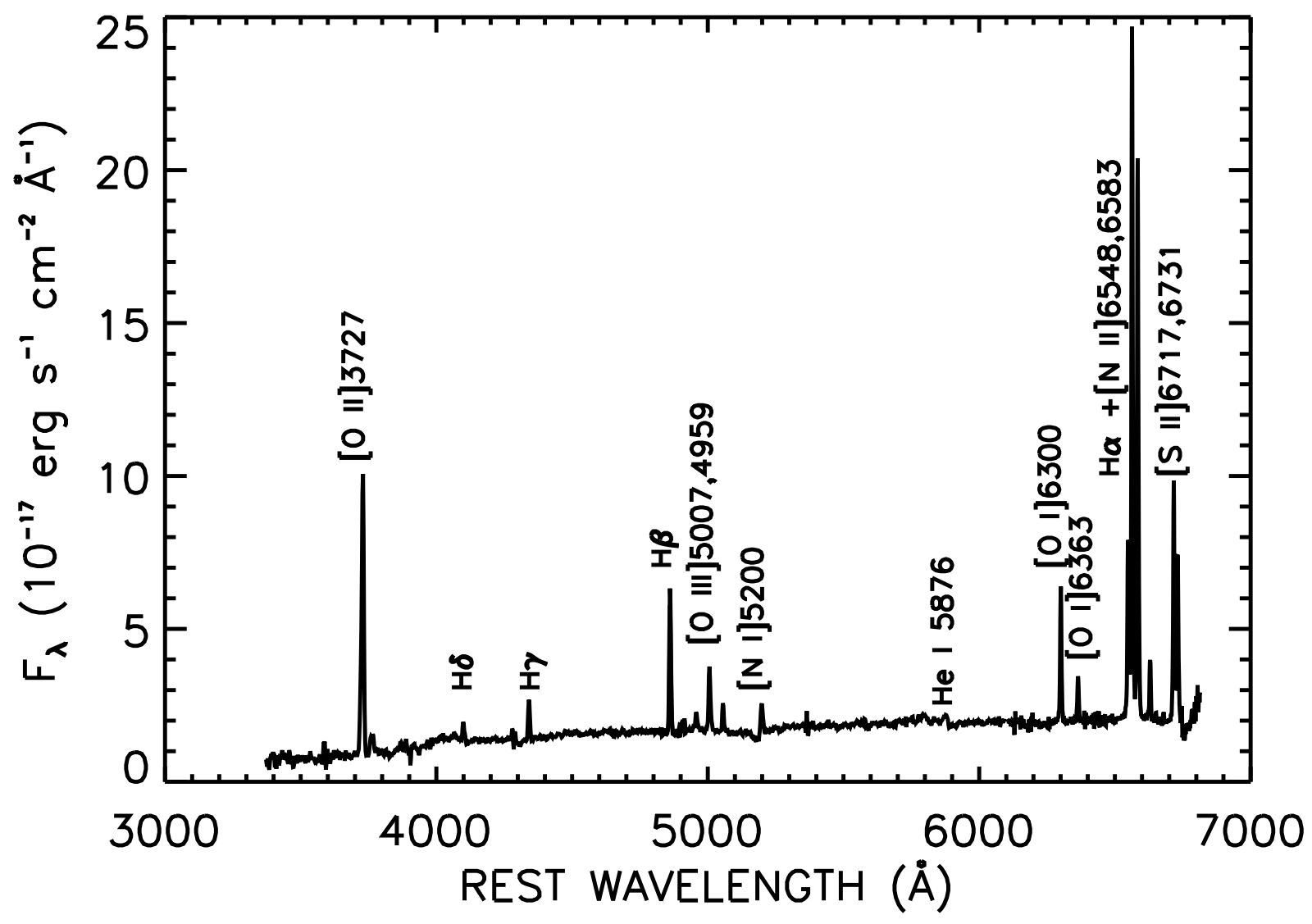

Fig. 2.- Composite spectrum generated from averaging filament spectra obtained at PAs $62^{\circ}, 77^{\circ}$, and $283^{\circ}$. The emission features seen at $5057 \AA$ and $6629 \AA$ are [O III] $\lambda 5007$ and $\mathrm{H} \alpha$, respectively, from the high-velocity system, redshifted by $c z \approx 3000 \mathrm{~km} \mathrm{~s}^{-1}$ relative to the low-velocity system. 

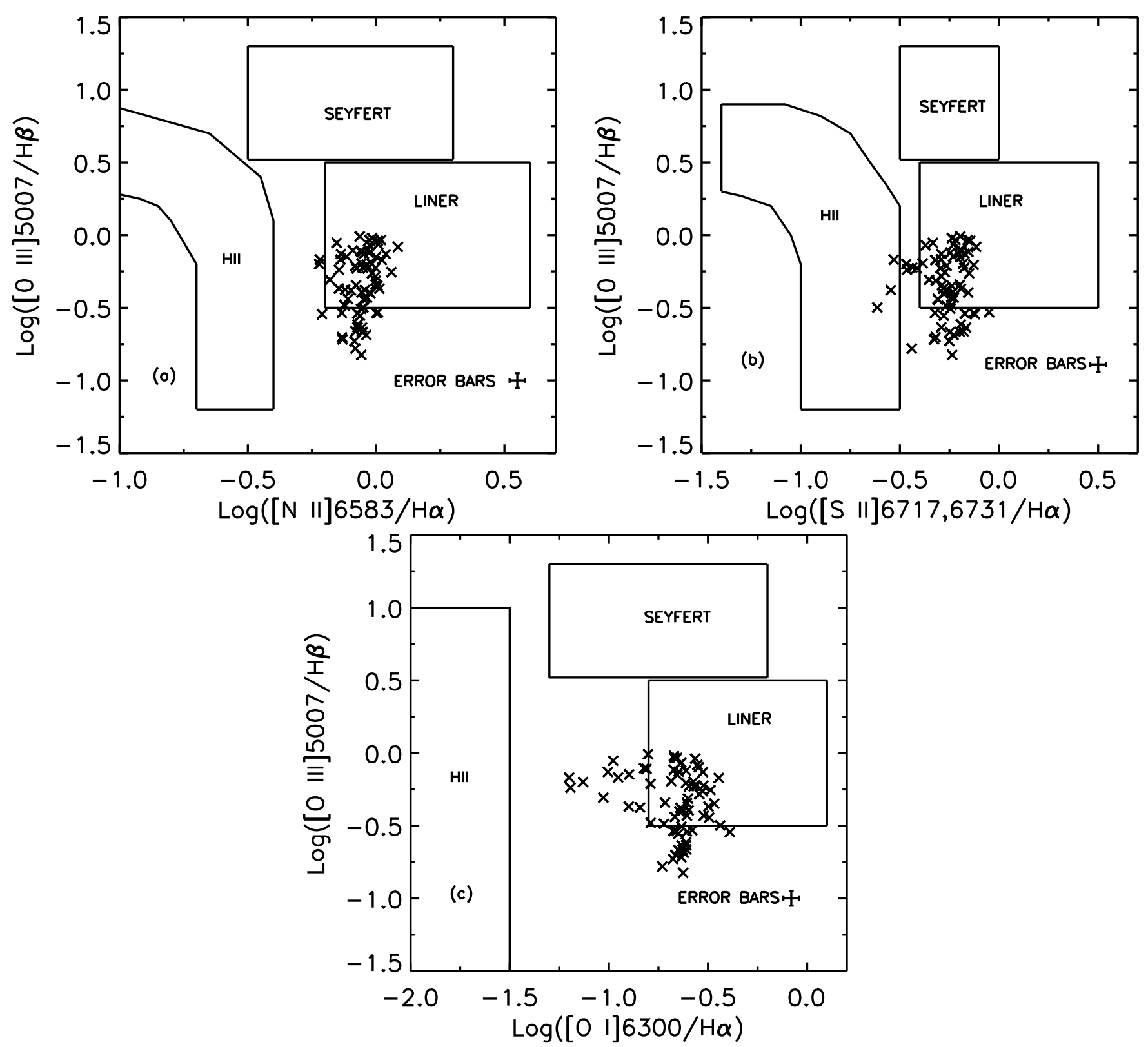

Fig. 3.- Line-ratio diagrams displaying reddening-corrected values measured at different locations within the filaments. Approximate loci employed for classification of nebular types are indicated. Representative $1 \sigma$ error bars are shown. 


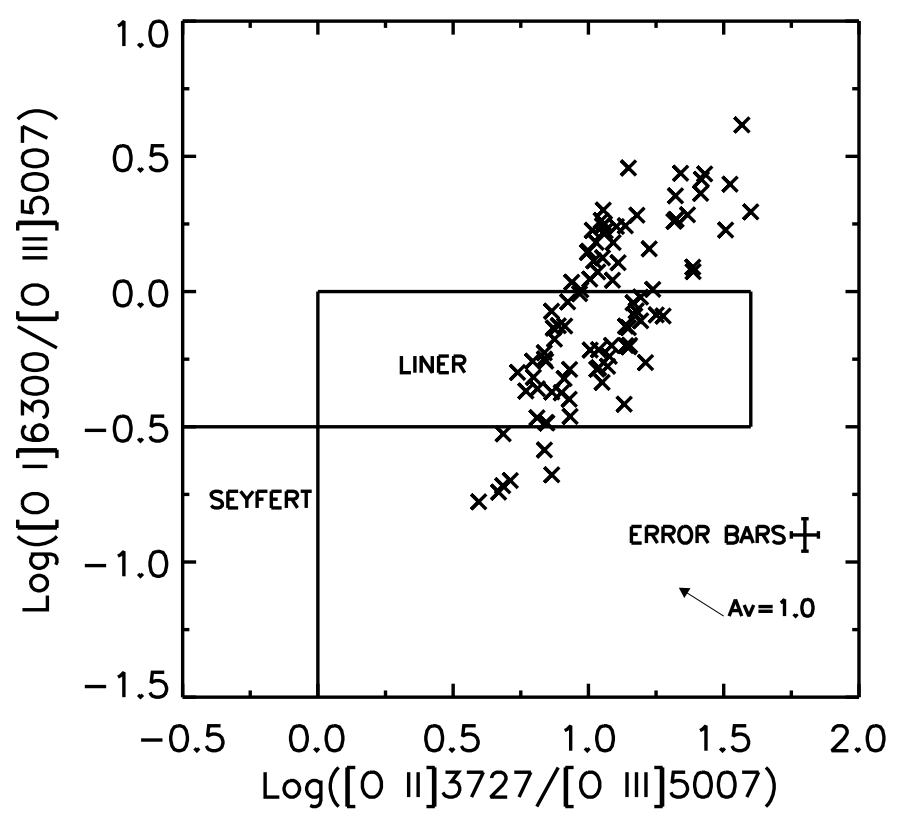

Fig. 4.- Line-ratio diagram employing lines of oxygen only, with reddening-corrected filament measurements shown. The effect of reddening by $A_{V}=1 \mathrm{mag}$ is given by the arrow. 

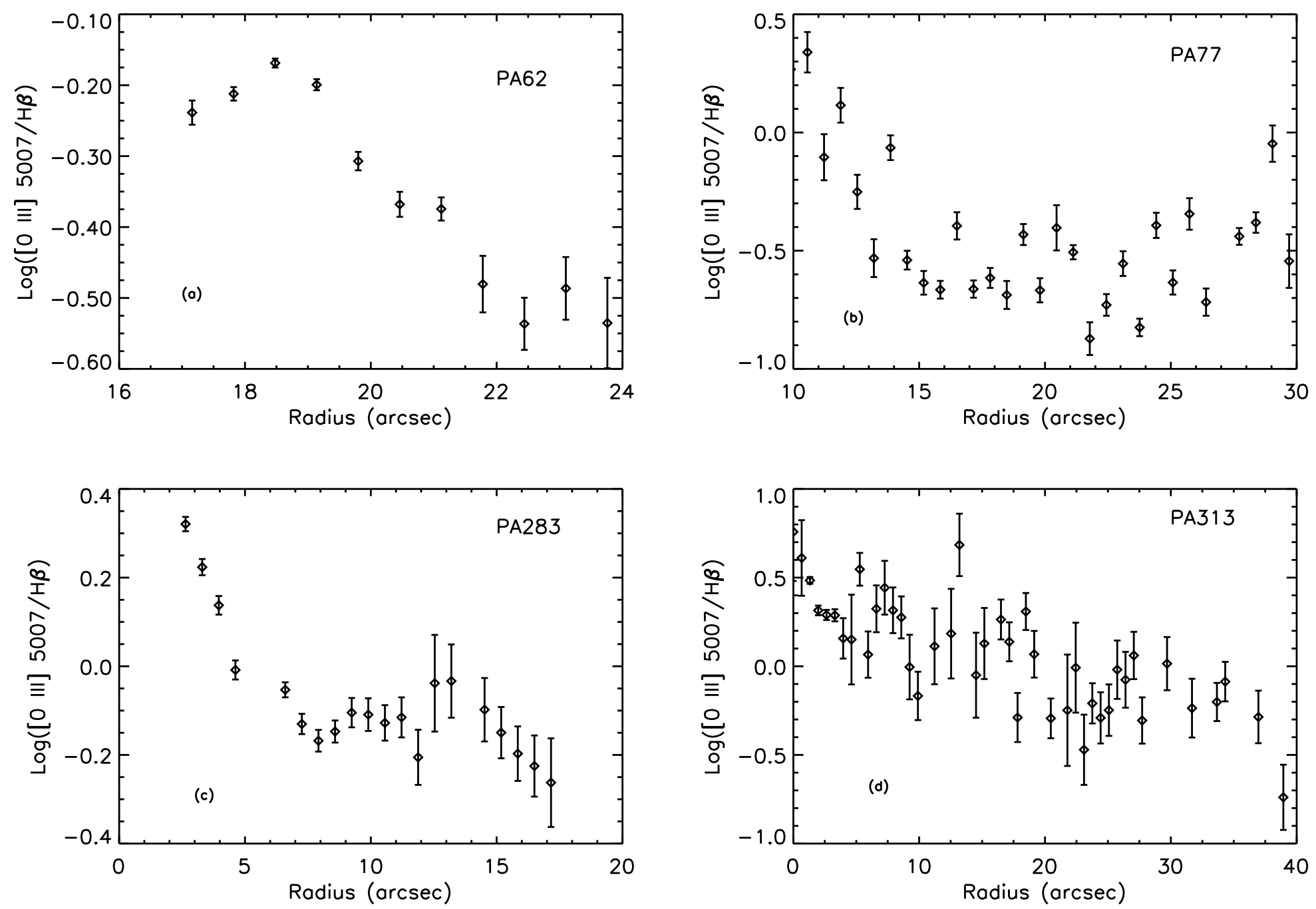

Fig. 5.- Radial profiles of the $[\mathrm{O} \mathrm{III}] / \mathrm{H} \beta$ line ratio at the four PAs; error bars represent $1 \sigma$ uncertainties. 


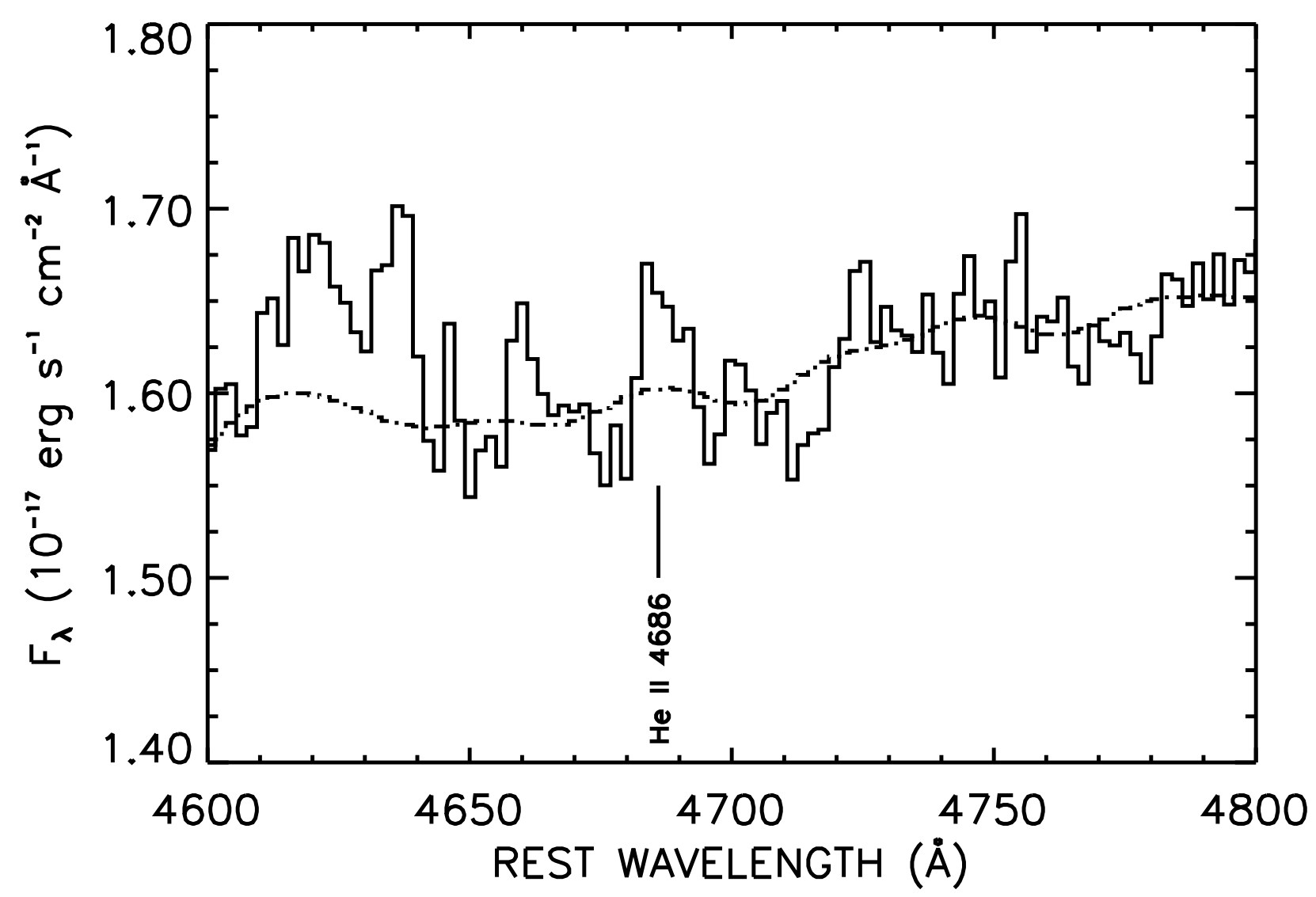

Fig. 6. - Expanded view of the composite spectrum from Figure 2, illustrating the measurement for NGC 1275 (solid histogram) and best-fitting continuum template (dashed line) in the vicinity of He II $\lambda 4686$. The expected location of this emission feature is shown. 


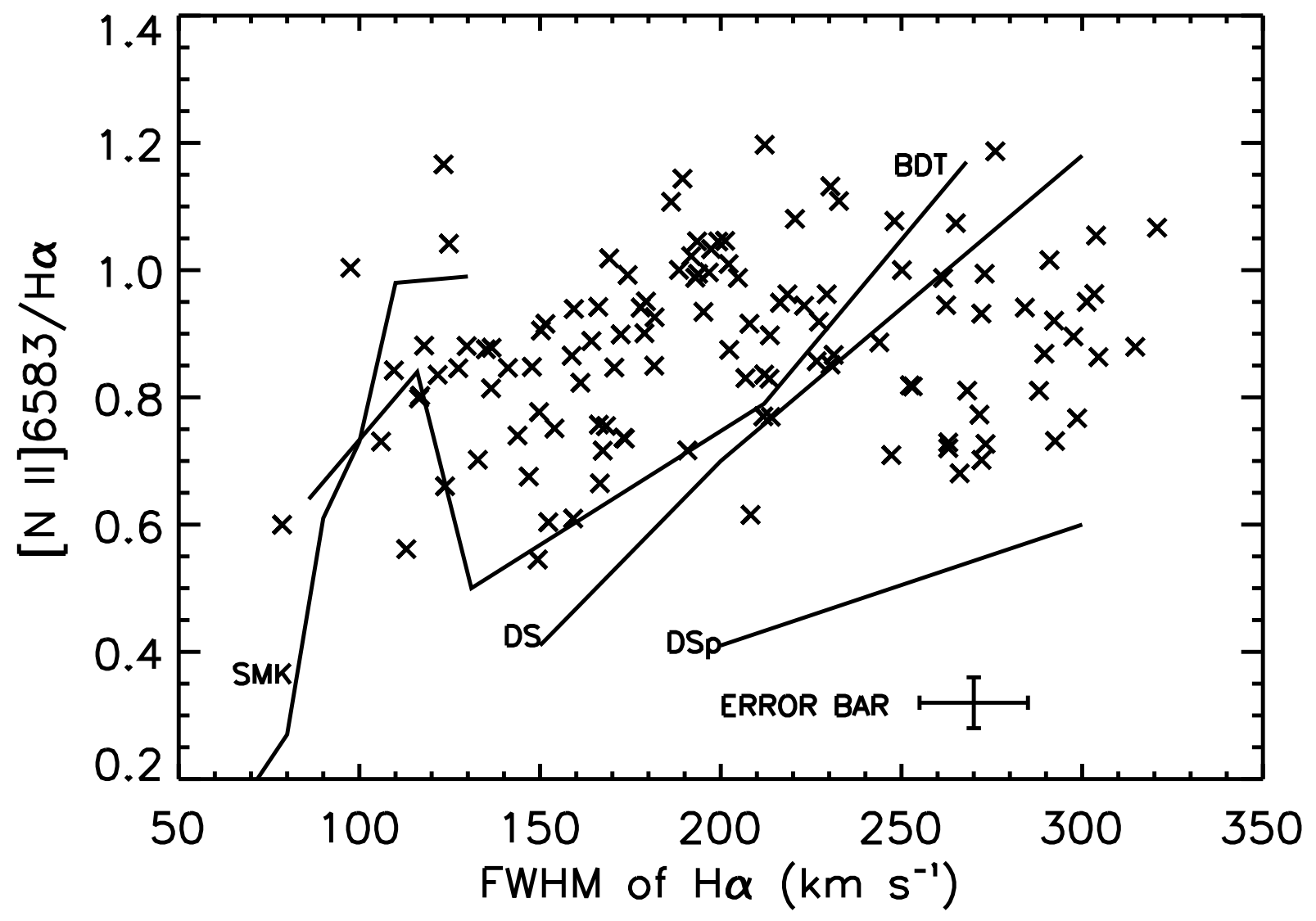

Fig. 7.- Reddening-corrected [N II] $\lambda 6583 / \mathrm{H} \alpha$ line ratio as a function of $\mathrm{H} \alpha$ line width for the filament measurements. Also shown by the solid lines are predictions of shock models taken from Dopita \& Sutherland (1995; DS = shock only, DSp = shock + precursor), Binette et al. (1985; BDT), and Shull \& McKee (1979; SMK). In plotting the model predictions, we have equated the shock velocity to the FWHM of $\mathrm{H} \alpha$. 


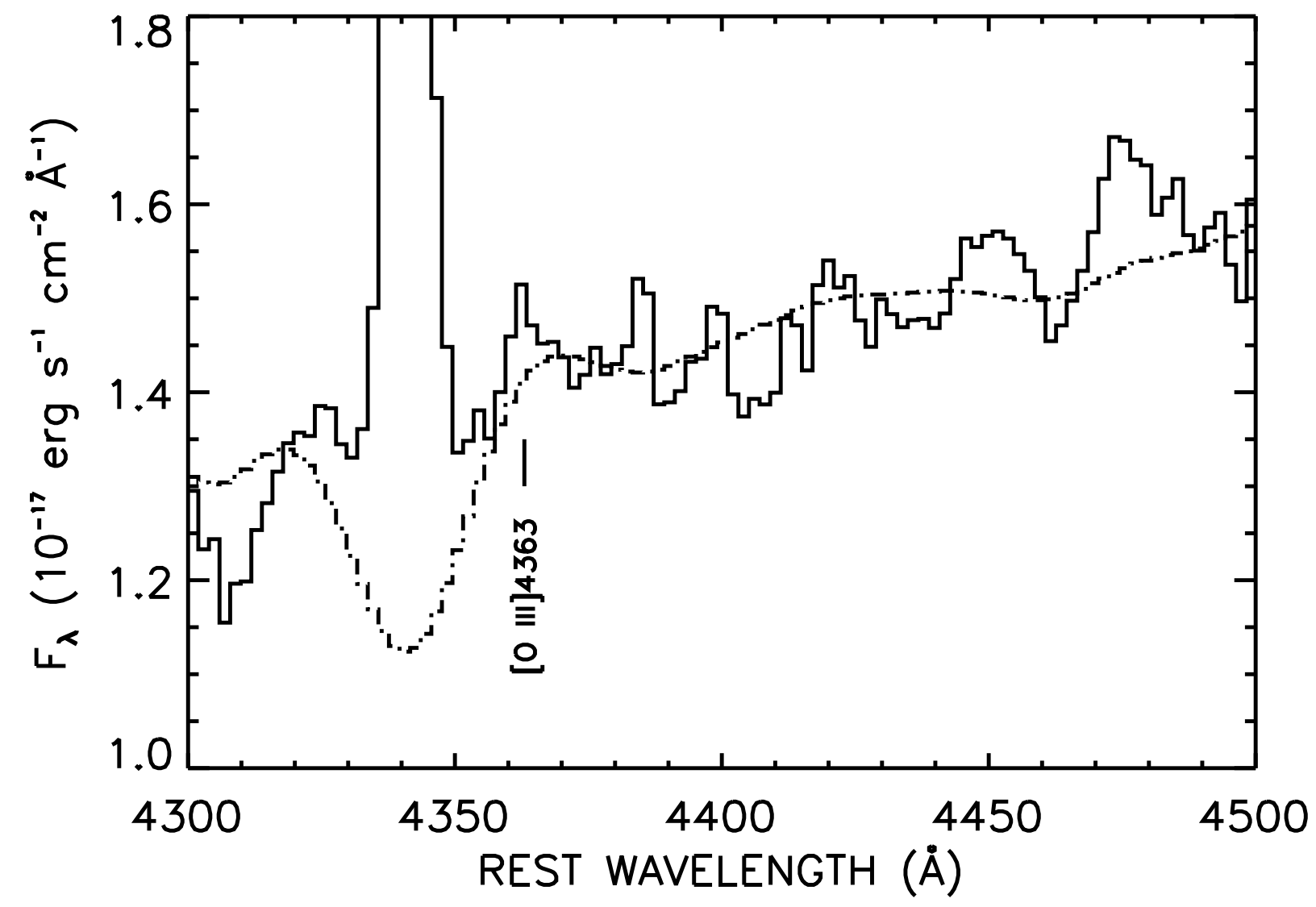

Fig. 8.- Similar to Figure 6, illustrating the spectral region around [O III $] \lambda 4363$. 


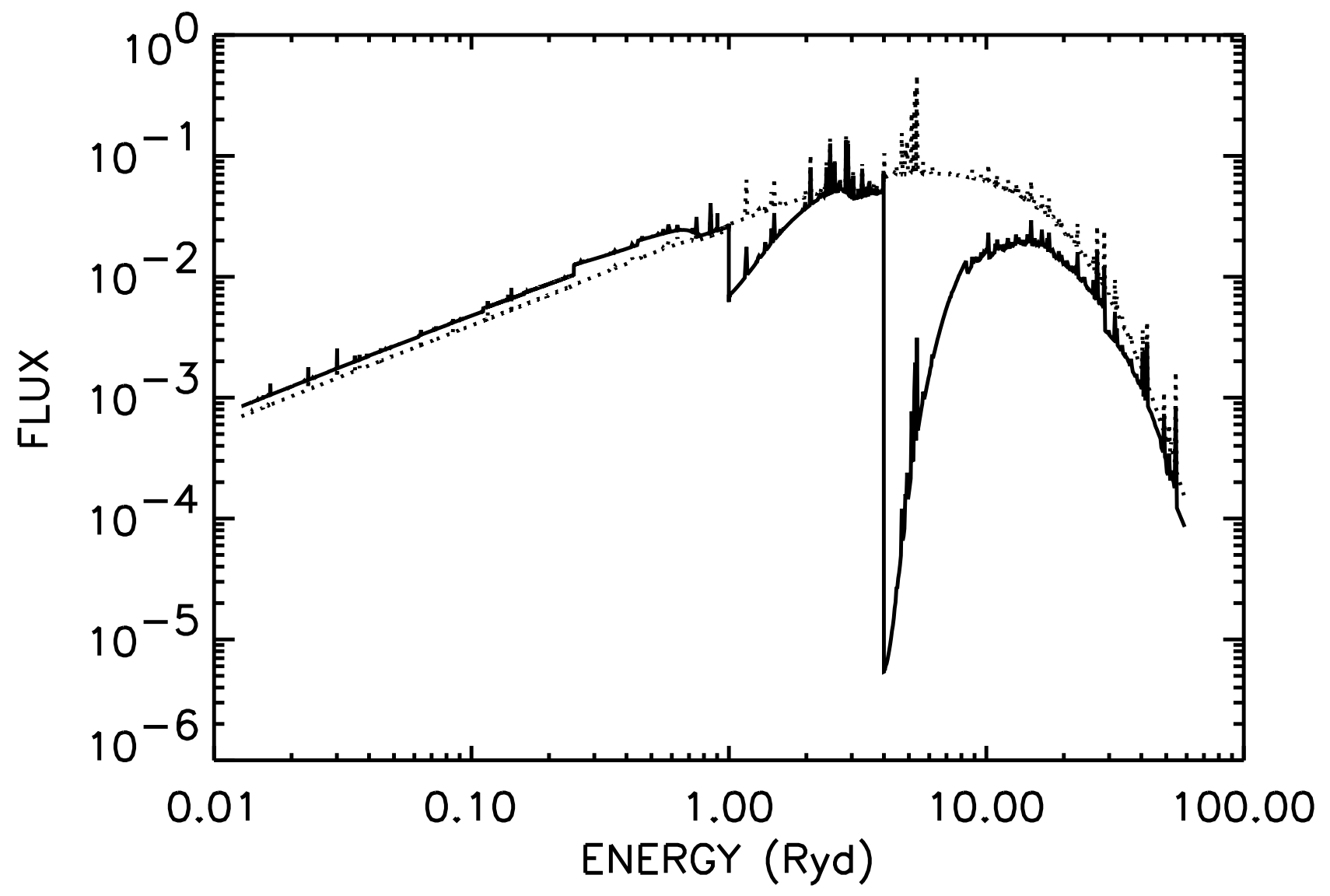

Fig. 9.- Model continua employed in photoionization calculations. The dotted line is the continuum incident on the screen, while the solid line is the transmitted continuum incident on the filament. Free-free, free-bound, and two-photon emission from the screen make the transmitted continuum higher than the incident one for energies below $\sim 0.8$ Ryd. 
Table 1: Observed Emission-Line Fluxes

\begin{tabular}{|c|c|c|}
\hline Emission Line & "Observed Flux ${ }^{1}$ & 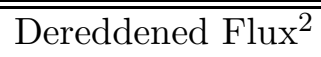 \\
\hline$[\mathrm{O}$ II $] \lambda 3727$ & $2.80 \pm 0.10$ & $4.80 \pm 0.17$ \\
\hline$[\mathrm{S} \mathrm{II}] \lambda 4068$ & $0.14 \pm 0.03$ & $0.19 \pm 0.04$ \\
\hline $\mathrm{H} \delta$ & $0.20 \pm 0.03$ & $0.27 \pm 0.04$ \\
\hline $\mathrm{H} \gamma$ & $0.35 \pm 0.04$ & $0.42 \pm 0.05$ \\
\hline$[\mathrm{O}$ III $] \lambda 4363$ & $<0.035$ & $<0.04$ \\
\hline He II $\lambda 4686$ & $<0.02$ & $<0.024$ \\
\hline $\mathrm{H} \beta$ & $1.00 \pm 0.03$ & $1.00 \pm 0.03$ \\
\hline$[\mathrm{O}$ III] $] \lambda 4959$ & $0.24 \pm 0.03$ & $0.23 \pm 0.03$ \\
\hline$[\mathrm{O}$ III $] \lambda 5007$ & $0.66 \pm 0.04$ & $0.62 \pm 0.04$ \\
\hline$[\mathrm{N} \mathrm{I}] \lambda 5200$ & $0.33 \pm 0.03$ & $0.30 \pm 0.03$ \\
\hline He I $\lambda 5876$ & $0.25 \pm 0.02$ & $0.19 \pm 0.02$ \\
\hline$[\mathrm{O}$ I $] \lambda 6300$ & $0.82 \pm 0.04$ & $0.59 \pm 0.03$ \\
\hline$[\mathrm{O}$ I $] \lambda 6364$ & $0.33 \pm 0.04$ & $0.24 \pm 0.03$ \\
\hline$[\mathrm{N}$ II $] \lambda 6548$ & $1.22 \pm 0.06$ & $0.84 \pm 0.04$ \\
\hline $\mathrm{H} \alpha$ & $4.15 \pm 0.14$ & $2.86 \pm 0.10$ \\
\hline$[\mathrm{N} \mathrm{II}] \lambda 6583$ & $3.54 \pm 0.12$ & $2.43 \pm 0.08$ \\
\hline He I $\lambda 6678$ & $0.07 \pm 0.03$ & $0.05 \pm 0.02$ \\
\hline$[\mathrm{S} \mathrm{II}] \lambda 6717$ & $1.38 \pm 0.06$ & $0.91 \pm 0.04$ \\
\hline$[\mathrm{S} \mathrm{II}] \lambda 6731$ & $1.07 \pm 0.05$ & $0.70 \pm 0.03$ \\
\hline
\end{tabular}

${ }^{1}$ Normalized to $\mathrm{H} \beta=1.93 \times 10^{-16} \mathrm{erg} \mathrm{s}^{-1} \mathrm{~cm}^{-2} \operatorname{arcsec}^{-2}$.

2 Normalized to $\mathrm{H} \beta=7.11 \times 10^{-16} \mathrm{erg} \mathrm{s}^{-1} \mathrm{~cm}^{-2} \operatorname{arcsec}^{-2}$, after correction for $A_{V}=1.2 \mathrm{mag}$. 
Table 2: Upper Limits on Coronal Lines and Theoretical Predictions

\begin{tabular}{cccc}
\hline \hline Line $/ \mathrm{H} \alpha^{1}$ & Upper Limit $^{2}$ & Model $^{3}$ & Notes \\
\hline$[\mathrm{Ni}$ XII $] \lambda 4232$ & $1.8 \times 10^{-2}$ & $1.2 \times 10^{-4}$ & \\
{$[\mathrm{Ar}$ XIV $] \lambda 4414$} & $1.0 \times 10^{-2}$ & $1.0 \times 10^{-3}$ & 4 \\
{$[\mathrm{Fe} \mathrm{XIV}] \lambda 5303$} & $9.4 \times 10^{-3}$ & $1.6 \times 10^{-3}$ & 5 \\
{$[\mathrm{Ca}$ XV $] \lambda 5445$} & $1.5 \times 10^{-2}$ & $0.6 \times 10^{-3}$ & \\
{$[\mathrm{Fe} \mathrm{X}] \lambda 6374$} & $1.4 \times 10^{-2}$ & $2.1 \times 10^{-3}$ & \\
{$[\mathrm{Ni} X V] \lambda 6702$} & $7.9 \times 10^{-3}$ & $0.1 \times 10^{-3}$ & \\
\hline
\end{tabular}

${ }^{1}$ Surface brightness of $\mathrm{H} \alpha$ is $1.9 \times 10^{-15} \mathrm{erg} \mathrm{s}^{-1} \mathrm{~cm}^{-2} \operatorname{arcsec}^{-2}$, after correction for $A_{V}=1.2$ mag.

${ }^{2}$ After correction for $A_{V}=1.2$ mag.

${ }^{3}$ From Sarazin \& Graney (1991), unless otherwise noted.

${ }^{4}$ From Voit et al. (1994).

${ }^{5}$ Voit et al. (1994) predict $15.6 \times 10^{-3}$.

Table 3: Line Ratios of the Photoionization+Screen Model

\begin{tabular}{ccc}
\hline \hline Line Ratio & Photoionization Model & Observed Range \\
\hline He II $\lambda 4686 / \mathrm{H} \beta$ & 0.024 & $<0.024$ \\
He I $\lambda 5876 / \mathrm{H} \beta$ & 0.18 & $0.16-0.20$ \\
{$[\mathrm{O} \mathrm{I}] \lambda 6300 / \mathrm{H} \beta$} & 0.78 & $0.33-0.78$ \\
{$[\mathrm{~N}$ II $] \lambda 6583 / \mathrm{H} \beta$} & 2.59 & $2.10-3.00$ \\
{$[\mathrm{~S} \mathrm{II}] \lambda 6717,6731 / \mathrm{H} \beta$} & 3.00 & $1.38-1.83$ \\
{$[\mathrm{O}$ III $] \lambda 5007 / \mathrm{H} \beta$} & 0.43 & $0.28-0.63$ \\
\hline
\end{tabular}

